\title{
UMA ANÁLISE DE CITAÇÕES E CO-CITAÇÕES EM ESTRATÉGIA DE OPERAÇÕES EM SERVIÇO: UM ESTUDO DAS EMPRESAS DE PROJETOS
}

\section{A CITATION AND CO-CITATION ANALYSIS IN SERVICES OPERATIONS STRATEGY: A STUDY IN PROJECT BASED ENTERPRISES}

\author{
Juliano Munik $^{1}$; Edson Pinheiro de Lima ${ }^{2}$; Sérgio Eduardo Gouvêa da Costa $^{3}$ \\ ${ }^{1}$ Pontifícia Universidade Católica do Paraná - PUCPR - Curitiba - Brasil \\ juliano.munik@pucpr.br \\ ${ }^{2}$ Pontifícia Universidade Católica do Paraná - PUCPR - Curitiba - Brasil \\ Universidade Tecnológica Federal do Paraná - UTFPR - Curitiba - Brasil \\ e.pinheiro@pucpr.br \\ ${ }^{3}$ Pontifícia Universidade Católica do Paraná - PUCPR - Curitiba - Brasil \\ Universidade Tecnológica Federal do Paraná - UTFPR - Curitiba - Brasil \\ s.gouvea@pucpr.br
}

\begin{abstract}
Resumo
A Gestão Estratégica de Operações tem como objetivo contribuir para a manutenção do posicionamento competitivo das empresas em seus mercados de atuação, atendendo as necessidades dos clientes e gerando vantagens competitivas em relação aos concorrentes. Com a evolução dos mercados, alguns setores iniciam um processo de transformação, como exemplo, as modificações ocorridas no setor de Serviços onde, nos últimos anos, a sua participação na economia dos países tem crescido. Este artigo tem como objetivo avaliar a produção científica em Gestão Estratégica de Serviço, particularmente focando estudos realizados com empresas que trabalham por projetos. A estratégia de pesquisa fundamenta-se na análise via Redes Sociais, onde é possível mapear relações entre grupo de autores, palavras-chave e periódicos. A análise resulta no entendimento do atual estágio de desenvolvimento da pesquisa no tema e permite identificar oportunidades para a evolução dos estudos. O estudo identificou de uma forma objetiva, através de gráficos e tabelas quais os grupos de indivíduos, como autores e palavras-chave, apresentam maior influência na amostra pesquisada, guiando o pesquisador na construção de um entendimento acerca do estado da arte no assunto estudado, bem como a identificar os temas e tópicos mais relevantes para futuros trabalhos. Este estudo contribui para o desenvolvimento de aplicações relacionadas ao estudo de Redes Sociais, sendo possível através da análise dos dados, identificar a qualidade destas informações para futuros desenvolvimento da pesquisa.
\end{abstract}

Palavras-chave: gestão estratégica de operações, operações de serviço, organização por projetos, redes sociais. 


\section{Introdução}

As empresas têm como objetivo fundamental desenvolver estratégias que resultem no seu desenvolvimento competitivo o qual pode estar associado à manutenção de um determinado posicionamento estratégico, ou ao aumento da sua participação em um determinado mercado, também a diferenciação de seus produtos e serviços, a melhoria da sua eficiência operacional, ou ainda dentre outras possibilidades, uma melhor compreensão do ambiente em que a organização está inserida e dos diferentes stakeholders que nele atuam.

Segundo Neely e Jarrar (2004), desenvolver processos ligados a gestão estratégica de operações aumenta as chances de se alcançar sucesso na realização da estratégia empresarial. Machuca et al (2006) descrevem a importância do desenvolvimento da gestão estratégica de operações nas empresas, com objetivos relacionados ao aumento da competitividade, acompanhamento da evolução do mercado e das necessidades dos clientes. Já Hayes e Upton (1998) conceituam a gestão estratégica de operações como o alicerce para estratégias defensivas ou reativas, como as de ataque ou pró-ativas, construindo uma representação baseada na lógica de um jogo de xadrez.

Com a evolução dos mercados, alguns setores iniciam um profundo processo de transformação. Como exemplo, pode-se citar as modificações ocorridas no setor de Serviços, no qual os últimos anos a sua participação na economia dos países tem crescido. Servitização (servitization) é o termo utilizado para descrever a importância e a tendência que as operações baseadas em serviços têm para a economia mundial, ou seja, a desmaterialização da economia. De acordo com Ren e Gregory (2007), a ‘servitização’ é um processo no qual as empresas oferecem um pacote de serviços mais detalhado aos seus clientes, ampliando opções e melhorando a qualidade percebida por meio de soluções integradas. Para Neely (2007), as empresas de manufatura que buscam melhorar o desempenho em seus mercados de atuação, devem expandir suas atividades na forma de serviços.

Segundo Gianesi e Correa (1994), os serviços têm apresentado um aumento constante, demandando estudos mais específicos que caracterizem a evolução das pesquisas nessa área. Para Araújo e Springer (2006), os serviços são processos relacionados diretamente aos produtos, reduzindo incertezas envolvidas aos processos de fabricação. Brax (2005) cita que os processos de vendas, atendimento aos clientes e o desenvolvimento de oportunidades em novos mercados são processos relacionados diretamente a serviços internos nas empresas. Hill et al (2002) delimitam os estudos para a 'Gestão de Operações em Serviço' para assuntos sobre previsão, qualidade, desenvolvendo fornecedores e clientes. 
Algumas empresas têm seus modelos de gestão e produção organizados na forma de projetos. A obtenção de um bom resultado na realização deles, não depende apenas da adoção de boas práticas de gerenciamento dos mesmos, mas também do alinhamento das políticas desenvolvidas no nível da estratégia de operações com o modelo de gestão de projetos adotado. Para Shenhar e Dvir (2007) gerir projetos é adaptar processos, desenvolver novas ferramentas de apoio, treinar pessoas visando aumentar as chances de resultados satisfatórios para a organização em determinado empreendimento. Andersen (2006) destaca que a gestão de projetos está condicionada a um contexto estratégico que se estabelece no nível do negócio e de diferentes funções como RH, finanças, comercial e operações dentre outras.

Esta pesquisa tem como foco os sistemas de trabalhos em forma de projetos, com atuação nos setores de prestação de serviço. Para cada tipo de projeto existe a necessidade de um tipo de modelo de gerenciamento específico, pesquisando a necessidade de novas informações sobre este tipo de ambiente, relacionando com o ambiente das estratégias de operações de serviço.

O objetivo deste artigo é analisar o referencial teórico sobre gestão estratégica de operações e operações de serviço organizadas na forma de projeto, via construção de redes de relacionamento. Para Alejandro e Norman (2006), com tal técnica de análise pode-se observar a interação entre os autores, sendo possível obter resultados para análises tanto do tipo qualitativa, quanto quantitativa, utilizando-se para tanto gráficos, representação por redes e o cálculo de indicadores de relacionamento, permitindo analisar redes construídas e aspectos como: densidade, centralidade, centralização, proximidade e intermediação.

Este artigo está dividido em quatro partes, sendo a primeira caracterizada por um levantamento bibliográfico, a segunda explica o uso da metodologia de análise de redes sociais, para em seguida, em terceiro plano destaca-se a análise e discussão dos resultados levantados junto às bases de dados escolhidas. A conclusão do artigo, constitui a quarta parte, onde destacam-se a contribuição do trabalho para a pesquisa em gestão de operações e a identificação de oportunidades futuras de pesquisa.

\section{Metodologia de Pesquisa}

Este artigo tem como objetivo principal desenvolver uma análise de citação e co-citação, buscando dentro de um tema específico de pesquisa avaliar a contribuição dos principais autores, identificando os tópicos estudados e os meios de divulgação utilizados. Algumas informações como ano de publicação, periódicos, banco de dados, origem das publicações, autores e palavras-chave descrevem a massa de dados estudada e complementam o estudo de redes de relacionamento.

O crescimento e evolução das pesquisas na área de Gestão Estratégica de Operações e Serviço são analisados com o desenvolvimento deste trabalho de pesquisa. Nota-se, que o aumento 
continuo das publicações no decorrer dos anos é alimentado pelo interesse de diversos autores e acadêmicos, conforme as necessidades de evoluções das ferramentas, dos processos e das pesquisas que as organizações necessitam para manterem-se competitivas no mercado de atuação. A revisão da literatura utiliza 79 amostras de pesquisas, sendo artigos acadêmicos, livros e uma dissertação de mestrado, publicados entre o período de 1969 e 2010.

Para Seuring e Muller (2008), esse tipo de metodologia de pesquisa, faz uma revisão da literatura compreendendo uma análise quantitativa e qualitativa sobre os aspectos descritivos do assunto estudado. Mayring (2003), apresenta um modelo de análise para pesquisa bibliográfica, composto por quatro etapas, sendo a primeira o processo de coleta do material, delimitando a unidade de análise, a segunda etapa compreende o tipo de análise descritiva, em seguida os principais temas da pesquisa são analisados (objetivos propostos) e finalizando com a quarta etapa, o material analisado resulta na interpretação dos dados e resultados apresentados.

\subsection{Redes Sociais}

A análise proposta na forma de redes permite observar informações do tipo: como os autores e assuntos estão relacionados e interligados entre si, qual o número de citações de um determinado assunto, qual o período onde aconteceu o maior número de citações e a quantidade de autores que estudaram um determinado assunto em um período de tempo.

Para Alejandro e Norman (2006), as redes sociais são grupos de pessoas, indivíduos ou entidades que se relacionam entre si, com a troca mútua de um fluxo de informações. Segundo Capra (2003), a característica através da qual se identificam nas redes sociais é o estilo de comunicação envolvendo uma linguagem, uma limitação cultural e relações de poderes entre os indivíduos envolvidos.

Uma rede é composta por três elementos básicos: nós, vínculos e fluxos.

a) Nós: interação dos indivíduos que compõe a rede social, aproximação dos mesmos que como relacionamento entre eles.

b) Vínculos: são os laços que existem entre dois ou mais nós. Os vínculos entre um nó e outro são representados por linhas;

c) Fluxos: indicam a direção que a informação está sendo direcionada ou interligada, também indicada por uma linha e uma seta. Os fluxos podem ser unidirecionais quando a informação é destinada para um nó e bidirecional quando o fluxo de informações é transmitido e recebido pelo mesmo indivíduo ou nó.

Na Figura 1, apresentam-se os elementos básicos de uma rede social, conforme definido por Alejandro e Norman, (2006). 
Figura 1- Elementos básicos de uma rede de Interações

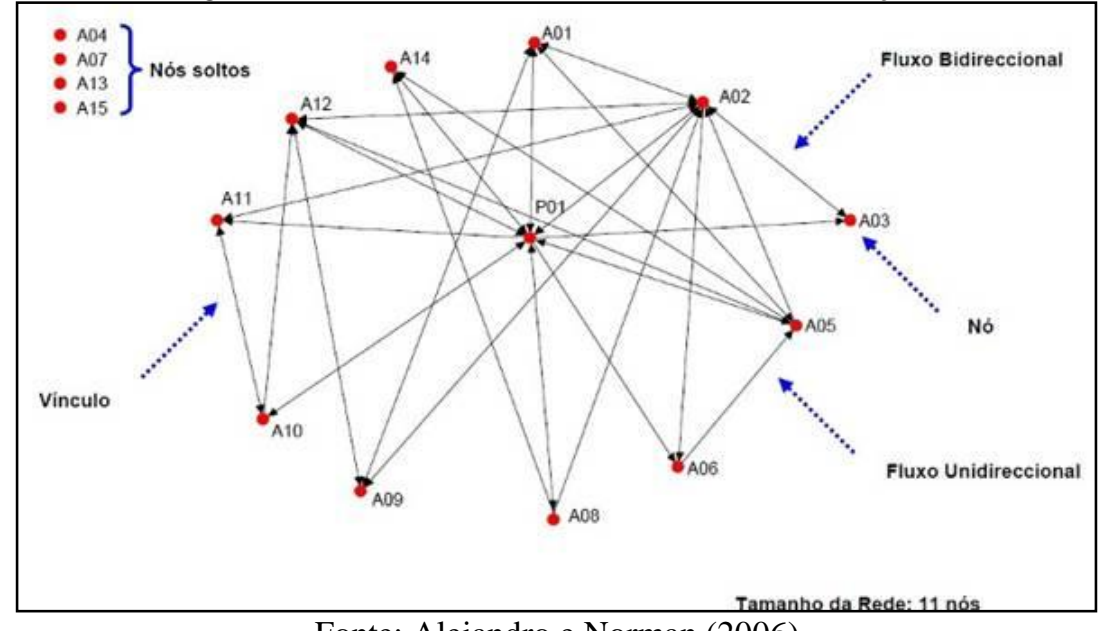

Fonte: Alejandro e Norman (2006)

Dados os elementos constitutivos de uma rede de relacionamentos, podem ser definidos os procedimentos de análise.

\subsection{Desenvolvimento do Procedimento de Análise}

Nesta seção do trabalho é apresentado todo o processo que culmina com a construção das redes de relacionamento. A pesquisa é operacionalizada pelo software UCINET 6.109®, particularmente gerando representações gráficas das redes, bem como estatísticas dos indicadores associados à análise de relacionamento. O processo está subdividido em quatro etapas principais, sendo a primeira, um estudo de natureza teórico conceitual. A segunda a definição da estratégia de pesquisa, para que na seqüência, na terceira, o planejamento da pesquisa possa ser elaborado. A quarta etapa consiste no desenvolvimento da pesquisa e da análise e interpretação dos dados.

No estudo de natureza teórico-conceitual é realizada a identificação do assunto principal da pesquisa, tendo como objetivo discutir aspectos conceituais da teoria. Esse processo permite que o autor obtenha mais clareza e formulação do problema de pesquisa, facilitando também a formulação das hipóteses e cumprimento dos demais objetivos da realização do trabalho. Com as informações desta etapa é possível perceber algumas discordâncias entre alguns pontos, apresentando novas propostas e identificação de novas áreas de pesquisa.

Será realizada uma descrição das características e relações das variáveis sobre o assunto estudado, como objetivo de compreender as características de um grupo, de uma comunidade. Enfatiza-se no estudo, o uso de redes sociais ou de relacionamento, particularmente aplicado a análise de citações e co-citações. O trabalho é voltado para uma análise de um conjunto de autores, referências e palavras-chave que apresentam relações e interações, sobre um determinado assunto abordado, como explicado no parágrafo anterior. 
A metodologia de análise através das Redes Sociais permite identificar como o grupo de autores e palavras chaves sendo o problema de pesquisa estudado, estão relacionados entre si, havendo uma troca de informações dentro de uma comunidade em comum. Essa metodologia orienta o pesquisador a direcionar a coleta de informações e busca dos artigos de um determinado assunto, sendo possível apresentar mais coerência e qualidade dos dados utilizados para o desenvolvimento da pesquisa.

O planejamento da pesquisa apresenta informações gerais sobre a estrutura de investigação, orientando o pesquisador a desenvolver o processo de amostragem, técnicas de coletas de dados, desenvolvimento dos dados e construção de planilhas eletrônicas e quadros descritivos que irão alimentar o software UCINET 6.109®.

Para o desenvolvimento desta análise foi utilizado uma amostra de 79 unidades, sendo composta majoritariamente por artigos acadêmicos. Tal amostra é o resultado da busca e pesquisa em bases de dados, através da utilização de um grupo de palavras-chave e assuntos pré-definidos pelos autores.

Quando iniciado o processo de busca dos artigos acadêmicos, livros e demais fontes de pesquisa, as informações foram catalogadas em uma planilha eletrônica do MS Excel ${ }^{\circledR}$ contendo dados sobre a área da pesquisa, a base de dados, ano de publicação, autores, local de publicação, países da publicação e palavras-chave que cada artigo contém, conforme apresentado na Tabela 1.

Tabela 1 - Catálogo de publicações

\begin{tabular}{|c|c|c|c|c|c|c|c|c|c|}
\hline Área & $\begin{array}{c}\text { Base } \\
\text { dados }\end{array}$ & Tipo & $\begin{array}{l}\text { Autores } \\
\text { citados }\end{array}$ & Referência & Título & Autor & Ano & $\begin{array}{c}\text { País } \\
\text { Publicação } \\
\end{array}$ & $\begin{array}{c}\text { Local } \\
\text { Publicação } \\
\end{array}$ \\
\hline $\begin{array}{c}\text { Gestão } \\
\text { 1. } \quad \text { Estratégica } \\
\text { de Operações }\end{array}$ & XX & artigo & 66 & 34 & XX & XX & 2004 & EUA & $X X$ \\
\hline $\begin{array}{c}\text { Operações de } \\
\text { Serviço }\end{array}$ & XXX & artigo & 40 & 31 & XXX & XXX & 1994 & Inglaterra & XXX \\
\hline
\end{tabular}

Fonte: Autores (2010)

A construção desta tabela tem como objetivo compilar as informações do levantamento bibliográfico e prepará-las para a construção da matriz de relacionamentos que constitui os dados de entrada para as redes de relacionamento.

Como o objetivo principal deste trabalho é identificar e analisar informações relacionadas de um grupo autores, referências e palavras-chave, após o desenvolvimento da planilha eletrônica em MS Excel ${ }^{\circledR}$, uma matriz é plotada com informações da quantidade de citações que os autores realizaram dentro de um assunto de pesquisa. Essas citações são referências a outros autores e suas respectivas publicações, identificados na amostra de 79 unidades encontradas nas bases de dados.

Para o desenvolvimento de um gráfico de redes utilizando o software UCINET 6.109® como ferramenta de apoio, primeiramente é necessário a construção de uma matriz quadrada para 
alimentar as informações, que irão formar as redes. As matrizes devem ser desenvolvidas de tal forma a representar um sistema de fluxos unidirecionais, conforme a Figura 02 abaixo.

Figura 2 - Matriz utilizada para construção de Redes Sociais

\begin{tabular}{|c|c|c|c|c|c|c|c|c|c|c|c|c|c|c|c|c|c|c|c|c|}
\hline & 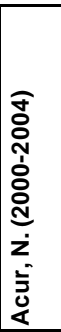 & 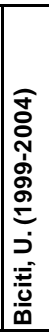 & 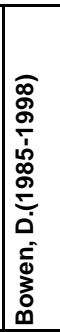 & 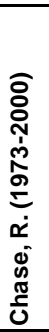 & 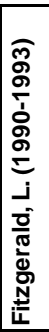 & 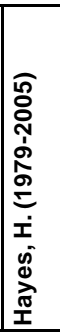 & 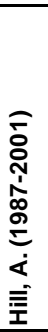 & 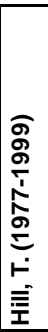 & 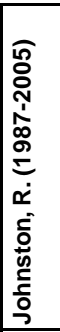 & 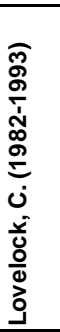 & 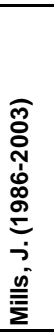 & 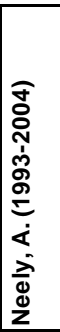 & 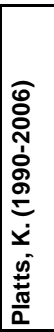 & 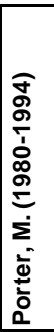 & 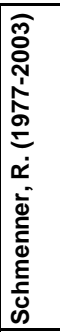 & 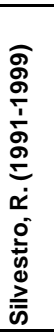 & 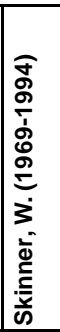 & 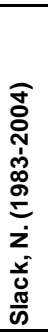 & 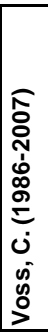 & 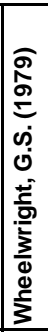 \\
\hline Acur, N. (2000-2004) & 6 & 7 & 0 & 0 & 0 & 6 & 0 & 1 & 0 & 0 & 3 & 3 & 5 & 2 & 0 & 0 & 2 & 2 & 1 & 3 \\
\hline Biciti, U. (1999-2004) & 4 & 5 & 0 & 0 & 0 & 5 & c & & 0 & 0 & 2 & 2 & s & 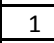 & 0 & & 1 & 2 & 1 & 2 \\
\hline Bowen, D.(1985-1998) & 0 & 0 & 6 & 2 & 0 & 0 & 0 & 0 & 0 & 2 & 0 & 0 & c & 0 & 0 & & 0 & 0 & 0 & 0 \\
\hline Chase, R. (1973-2000) & 0 & 0 & 1 & 3 & 5 & 2 & 0 & 2 & 6 & 1 & 0 & 0 & 0 & 0 & 1 & 4 & 0 & 2 & 4 & 2 \\
\hline Fitzgerald, L. (1990-1993) & 0 & 0 & 0 & 1 & 1 & 7 & 0 & 1 & 1 & 0 & 2 & 4 & 2 & 3 & 2 & 0 & 4 & 2 & 3 & 4 \\
\hline Hayes, H. (1979-2005) & 4 & 5 & 0 & 0 & 0 & 5 & 0 & 1 & 0 & 0 & 3 & 1 & 3 & 0 & 0 & 0 & 1 & 1 & 1 & 3 \\
\hline Hill, A. (1987-2001) & 0 & 0 & 0 & 5 & 1 & 2 & 8 & 0 & 2 & 1 & 0 & 0 & 0 & 1 & 2 & 1 & 1 & 0 & 4 & 2 \\
\hline Hill, T. (1977-1999) & 0 & 0 & 0 & 0 & 0 & 4 & 0 & 1 & 0 & 0 & 0 & 0 & 0 & 0 & 0 & 0 & 2 & 0 & 0 & 5 \\
\hline Johnston, R. (1987-2005) & 0 & 0 & 7 & 9 & 4 & 1 & 1 & 0 & 35 & 8 & 0 & 3 & 0 & 0 & 1 & 6 & 2 & 3 & 7 & 1 \\
\hline Lovelock, C. (1982-1993) & 0 & 0 & 4 & 2 & 0 & 0 & 0 & 0 & 0 & 10 & 0 & 0 & 0 & 0 & 0 & 0 & 1 & 0 & 0 & 0 \\
\hline Mills, J. (1986-2003) & 0 & 0 & 0 & 0 & 0 & 1 & 0 & 0 & 0 & 0 & 4 & 3 & 5 & 2 & 0 & 0 & 1 & 0 & 2 & 3 \\
\hline Neely, A. (1993-2004) & 0 & 0 & 0 & 0 & 0 & 0 & 0 & 0 & 0 & 0 & 1 & 1 & 3 & 0 & 0 & 0 & 1 & 0 & 2 & 3 \\
\hline Platts, K. (1990-2006) & 0 & 0 & 0 & 0 & 0 & 1 & 0 & 0 & 0 & 0 & 4 & 3 & 5 & 2 & 0 & 0 & 1 & 0 & 2 & 3 \\
\hline Porter, M. (1980-1994) & 0 & 0 & 0 & 2 & 0 & 2 & 0 & 2 & 0 & 0 & 0 & 0 & 0 & 2 & 0 & 0 & 0 & 0 & 0 & 2 \\
\hline Schmenner, R. (1977-2003) & 0 & 0 & 2 & 2 & 0 & 2 & 0 & 0 & 0 & 1 & 1 & 0 & 0 & 0 & 3 & 0 & 0 & 0 & 1 & 2 \\
\hline Silvestro, R. (1991-1999) & 0 & 0 & 2 & 3 & 3 & 2 & 0 & 2 & 10 & 2 & 0 & 0 & 0 & 0 & 1 & 3 & 0 & 2 & 4 & 2 \\
\hline Skinner, W. (1969-1994) & 0 & 0 & 2 & 2 & 2 & 3 & 0 & 0 & 0 & 0 & 0 & 0 & 0 & 0 & 0 & 0 & 1 & 1 & 0 & 3 \\
\hline Slack, N. (1983-2004) & 0 & 0 & 0 & 1 & 0 & 0 & 0 & 0 & 1 & 0 & 0 & 1 & 0 & 0 & 1 & 1 & 0 & 0 & 0 & 1 \\
\hline Voss, C. (1986-2007) & 0 & 0 & 0 & 0 & 0 & 1 & 0 & 2 & 0 & 0 & 1 & 2 & 2 & 1 & 0 & 0 & 3 & 1 & 6 & 1 \\
\hline Wheelwright, G.S. (1979) & 0 & 0 & 0 & 0 & 4 & 0 & 1 & 0 & 0 & 0 & 0 & 0 & 0 & 0 & 0 & 0 & 2 & 0 & 0 & 5 \\
\hline
\end{tabular}

Fonte: Autores (2010)

Quando uma matriz simétrica for desenvolvida, haverá a existência de fluxos bidirecionais. Quando houver a presença de fluxos unidirecionais, significa que houve apenas o direcionamento ou troca das informações entre um indivíduo para outro. Pode-se citar como exemplo, quando um autor realiza a citação de outro autor, havendo apenas o direcionamento das informações de uma parte para outra. No caso de fluxos bidirecionais, ocorre quando os dois indivíduos realizam trocas mútuas de informações entre si (Figura 1).

As matrizes podem ser desenvolvidas no próprio software UCINET 6.109® ou importadas de uma planilha eletrônica (e.g. MS Excel $\left.{ }^{\circledR}\right)$, onde apresentarão a relação e interação que um indivíduo apresenta sobre outro indivíduo (autores/referências/palavras-chave). Na Figura 2 apresenta-se a matriz de relações dos principais autores que mais contribuíram com citações na amostra de artigos utilizados na pesquisa realizada.

Para contabilizar os autores que mais contribuíram para o tema de pesquisa, isto é, os que mais receberam citações, foi desenvolvido um processo de contagem das citações (artigos e autores) que constituíam o referencial bibliográfico dos artigos e livros selecionados. 
A contagem é realizada manualmente, utilizando-se como ferramentas de apoio, planilhas eletrônicas especialmente desenvolvidas para armazenar as informações levantadas junto às bases de dados escolhidas. Para a análise e interpretação de dados são utilizados os recursos disponíveis no software UCINET 6.109®, para a representação de redes e síntese de indicadores de relacionamento, bem como uma estatística descritiva da amostra de trabalhos selecionados. A Figura 3 apresenta o processo a ser seguido, para a construção da rede de relacionamentos, aplicada a análise de citações e co-citações.

Figura 3 - Metodologia para Construção de Redes Sociais

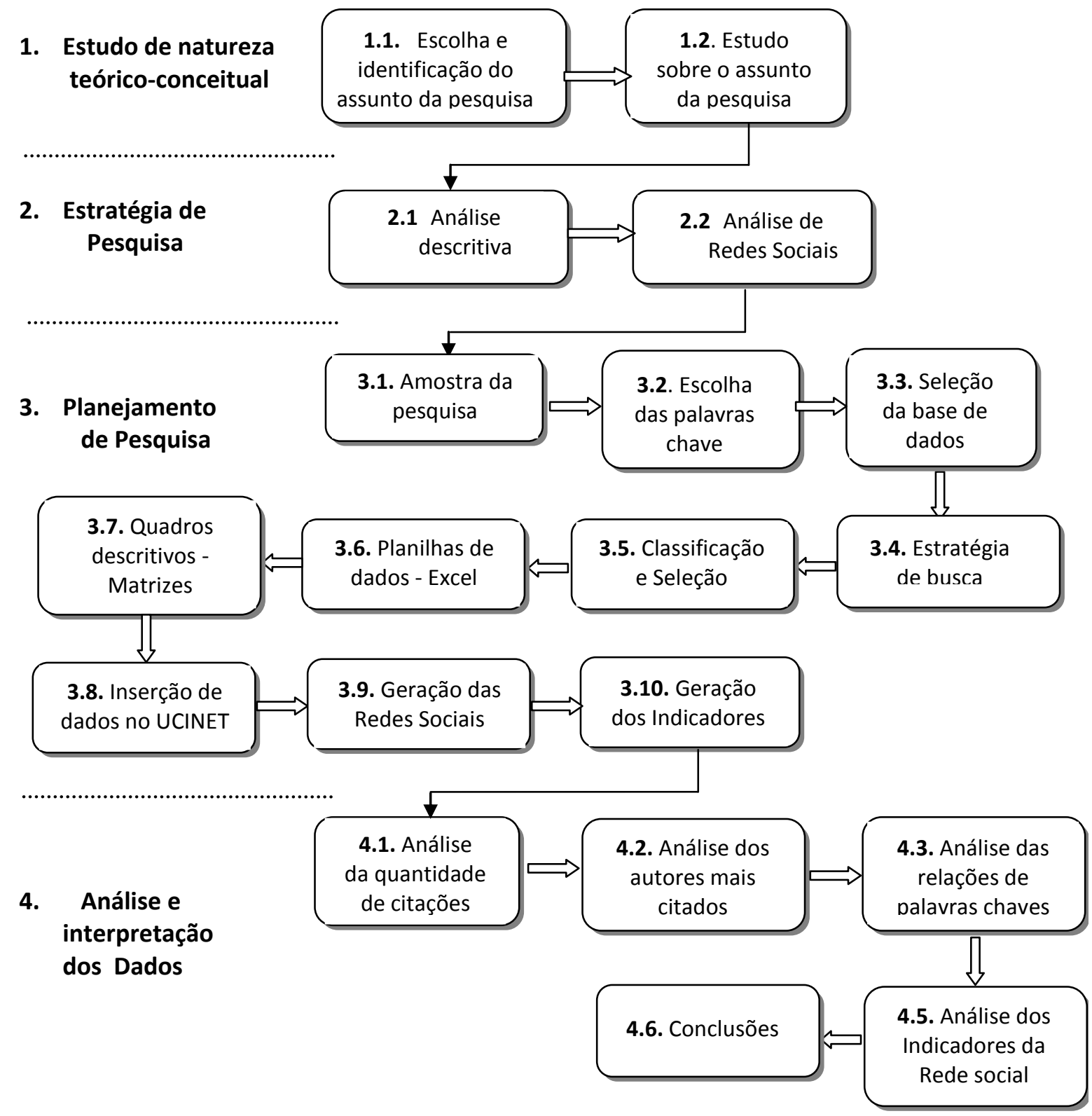

Fonte: Autores (2010)

Iniciando com o estudo da natureza teórico conceitual, sendo a etapa de identificação e estudos do assunto que será desenvolvido a pesquisa. Na etapa 02 é definida a estratégia de pesquisa, sendo importante definir o método de desenvolvimento do trabalho. Em seguida, na etapa 
03 é definido o planejamento de pesquisa, determinando-se a amostra pesquisada, bancos de dados a serem pesquisados, desenvolvimento das planilhas, geração e desenvolvimento dos indicadores com a utilização do software UCINET 6.109®. Finalizando com a etapa 04, obtém-se a análise dos resultados, através do desenvolvimento da discussão e conclusão do trabalho.

\subsection{Indicadores de Relacionamento}

Dentre as funcionalidades disponíveis no software UCINET 6.109®, há o estudo de indicadores de relacionamento, cobrindo os seguintes aspectos:

a) Densidade: apresenta o grau de conectividade que o nó tem dentro da rede;

b) Centralidade: número de relações existentes entre um indivíduo e outro;

c) Centralização: nível de centralização que um indivíduo apresenta na rede, sendo o maior fluxo de informações direcionado para esse mesmo indivíduo;

d) Intermediação: possibilidade que um indivíduo tem para intermediar um fluxo de informação;

e) Proximidade: capacidade que um indivíduo tem para se relacionar com todos ou o maior número de indivíduos da rede.

O software UCINET 6.109®, apresenta indicadores que podem ser utilizados para análises quantitativas e qualitativas, para o estudo de qualquer área da ciência. Como exemplo, Abreu e Pinho (2008), realizaram estudos e análises de indicadores relacionados a questões sociais e políticas, utilizados para a identificação de agentes influenciadores e formação dentro de uma Plataforma de um Movimento Social.

\section{Referencial Teórico}

A estrutura do referencial teórico esta divida em três seções. A primeira seção apresenta conceitos gerais sobre gestão estratégica de operações (GEO), abordando a sua importância nas organizações. Prosseguindo, a segunda aborda assuntos relacionados a operações de serviço, com informações sobre a definição e metodologias de implementação de estratégias nessa área. Finalizando, na terceira seção, desenvolve-se uma abordagem sobre organização das operações por projetos, com definições e utilizações de metodologias para gerenciamento dessas operações, onde exigem processos específicos para cada tipo de projeto.

O referencial teórico aborda um contexto para a análise dos resultados, através de quadros, tabelas e gráficos, sendo as informações geradas pela teoria de Redes Sociais. Essa análise será contextualizada através do embasamento teórico, sendo o objetivo deste trabalho o estudo e levantamento bibliográfico de algumas áreas relacionadas a Gestão Estratégica de Operações, Serviços e Organizações por Projetos. 


\subsection{Gestão Estratégica de Operações}

A GEO tem como objetivo estabelecer critérios para o desenvolvimento de metas e otimização dos resultados da empresa, atendendo às necessidades dos clientes e mantendo o posicionamento da empresa dentro de seu mercado de atuação.

Trabalhos como os de Hayes e Pisano (1994) e Hayes e Upton (1998) contribuí sobremaneira para a evolução das pesquisas relacionada a gestão estratégica de operações, destacando a importância deste enfoque no desenvolvimento estratégico das empresas. A GEO vem constituindo um dos temas de interesse na agenda de pesquisa de publicações como a Decision Sciences há quase três décadas (MILLER, et al 1981). Pesquisas subseqüentes, como as de Gyampah e Meredith (1989), Pilkington e Liston-Heyes (1999), Pilkington e Fitzgerald (2006), Taylor e Taylor (2009) e Pilkington e Meredith (2009) apresentam a evolução dos estudos em GEO, no contexto da gestão de operações, que nitidamente evoluíram de uma visão funcional e instrumental para uma visão de natureza sistêmica e estratégica.

Para Hill (1993), a GEO é uma abordagem visando o aumento da vantagem competitiva, respondendo as exigências do mercado e dos clientes. Abordagens baseadas no uso estratégico de tecnologia, recursos, processos e competências são importantes para os sistemas de operações, pois se desenvolvem na forma de um processo de aprendizagem resultando em melhoria contínua e aumento da competitividade. Wheelwright (1984), define a GEO na medida da relação entre a estratégia de operações e a estratégia de negócios da empresa em todos os seus aspectos, enquanto que Voss (1995), destaca a importância para a forma de relacionamento entre estratégias, a família de produtos e as linhas produtivas, sob a ótica de satisfação dos clientes.

As estratégias quando desenvolvidas por meio de um processo sistematizado e de acordo com um determinado modelo, podem aumentar a probabilidade de sucesso nos resultados das empresas, contribuindo para o alinhamento interno e externo dos sistemas de planejamento (SKINNER, 1974). A utilização formal de planos estratégicos, visando o aumento de vantagens competitivas, pode ser materializada na forma de sistemas que organizam 'decisões' e 'ações', aumentando as chances das organizações conseguirem seus objetivos de longo prazo (SLACK, et al 1997). Observa-se que ao longo deste processo as competências desenvolvem-se, ampliando continuamente a capacidade de realização da estratégia e o leque de opções estratégicas (BOWEN, et al 1990).

Platts et al (1996) definem que a competitividade da empresa depende do resultado da capacidade de escolher os objetivos estratégicos do negócio e das operações com base no mercado. 
As empresas devem centrar-se no desenvolvimento de uma estratégia 'correta' e específica, com uma visão abrangente da interação desta mesma estratégia com seu mercado específico de atuação.

Segundo Hayes e Wheelwright (1984), a GEO busca alinhamento estratégico entre o nível da unidade de negócios e o nível da função operações, atendendo requisitos do cliente no mercado servido. Aspectos como qualidade, confiabilidade e custo, definem tais demandas em termos de produto.

O desenvolvimento da GEO acontece na interação entre uma visão de operações orientada a mercado e outra visão baseada em recursos, inter-relacionando aspectos externos e internos para aumentar o desempenho do negócio (CAMPBELL e LUCHS, 1997).

A GEO necessita de um processo sistematizado para sua aplicação nos ambientes empresariais. Procedimentos formalizados devem ser desenvolvidos de acordo com um modelo, com um processo lógico passível de ajustes conforme as mudanças dos cenários, mercados e objetivos pretendidos, garantindo a competitividade da empresa frente aos seus concorrentes.

Slack e Lewis (2002), apresentam um modelo que atende outro tipo de sistematização para o desenvolvimento de estratégias de operações. O modelo oferece o desdobramento da estratégia na forma de um processo, fundamentando-se em informações do mercado, financeiras e nos objetivos estratégicos da organização.

\subsection{Operações de Serviços}

Zeithaml e Bitner (2003) definem 'serviço' como um conjunto de processos para atender às demandas e necessidade de clientes. Para Kotler (1998), os serviços estão relacionados a processos intangíveis que uma parte oferece a outra. Vargo e Lusch (2004) o definem como a aplicação de competências e habilidades, por meio de processos para atender as necessidades de um cliente, entidade ou a si próprio. Kotler (1999) destaca que as operações de serviço são resultado de atividades internas na relação entre fornecedor e cliente interno, visando atender necessidades do cliente final.

Segundo Gianesi e Correa (1994), as atividades relacionadas a serviços têm sua contribuição nas organizações, na medida em que constituem:

a) Diferencial competitivo: empresas de manufatura têm prestado serviços ao cliente para diferenciação de seus pacotes de produto.

b) Suporte a manufatura: diversas funções internas das empresas de manufatura são operações de serviço, como recursos humanos, manutenção e sistemas de informação.

c) Geradores de lucro: as operações de serviço, em diversas empresas de manufatura podem desenvolver-se além de suas funções tradicionais.

Conforme Rosen (1990), os serviços apresentam características tais como: 
a) Inseparabilidade da produção e consumo: a participação do consumidor é direta impactando no resultado final do serviço.

b) Intangibilidade: os serviços são conceitos intangíveis que os clientes podem sentir e perceber.

c) Pericibilidade: os serviços não podem ser estocados e armazenados e sendo assim, diferem quanto as políticas de qualidade e de previsões de vendas.

d) Heterogeneidade: o mesmo serviço pode variar de fornecedor para fornecedor, sendo a qualidade resultado da experiência adquirida.

A produtividade e eficácia nos serviços estão diretamente relacionadas com a concepção do serviço e do sistema para sua implementação (ROSEN, 1990). Desenvolver uma abordagem para resolver as questões relacionadas à melhoria da qualidade e a produtividade dos serviços, traz novas técnicas para o projeto de produtos e processos (SIDHARTHA e CEM, 2006).

Para Schmenner (1986), a visão voltada para resultados que os serviços podem apresentar, é algo a se considerar na GEO de serviços. Muitos dos serviços até pouco tempo, eram tidos como únicos e conseqüentemente não havendo uma variedade de técnicas, metodologias e ferramentas para otimizar a sua gestão.

Na década de 1980, notadamente, verificou-se o aumento da diversificação dos serviços. No entanto havia poucos estudos no tema, o que significava pouca comunicação e aprendizagem na área. Orientação era importante, ou seja, cada tipo de serviço associado a determinada indústria era tido como diferente (SILVESTRO, 1999).

Na década de 1990, a diversidade de pesquisas marcou a evolução dos estudos de GEO em serviços tem aumentado. O crescimento da contribuição dos serviços para a economia dos países foi o grande motor deste crescimento (VERMA, 2000). Para Killen e Hunt (2010), a utilização e evolução de metodologias para o desenvolvimento de estratégias ligadas a serviços vêm crescendo nos últimos anos.

\subsection{Organizações por Projetos}

Algumas empresas podem desenvolver as operações de serviço na forma de projetos. Cada projeto é único, contendo sua complexidade e demandas específicas, havendo necessidade de uma metodologia estruturada para o seu gerenciamento. Há uma diversidade de estudos que propõem diferentes modelos para a gestão de projetos, definindo abordagens para o desdobramento, processos para organização e gestão e técnicas específicas para programação, seguimento e avaliação (ANDERSEN, 2006).

O gerenciamento de projetos pode ser definido como a mobilização de uma série de atividades temporárias, cujo objetivo principal é a criação de um produto ou um serviço único. O 
termo temporário pode significar ter um ponto inicial de partida e um ponto final de término. $\mathrm{O}$ projeto é único, pelo fato que cada produto entregue em forma de projeto é diferenciado de outros, sendo que cada um apresenta uma complexidade e necessidades específicas, exigindo estilos diferenciados de liderança, orçamentos variados, requisitos e procedimentos diversos (PMBOK, 2004).

Um projeto pode ser diferenciado de várias formas. Podem ser classificados de acordo com o risco, o ambiente, a tecnologia, o tamanho, dentre muitos outros aspectos. Ao mesmo tempo em que existem tais diferenças, algumas características também são comuns, como o fato de se ter um objetivo a ser alcançado, a existência de prazo para o cumprimento das atividades, o seguimento de um cronograma, o orçamento direcionado e uma equipe alocada para a realização e o gerenciamento do projeto (SHENHAR e DVIR, 2007).

Para Schmenner (1986), a formulação de estratégias para operações ligadas a serviços na forma de projetos representa um conjunto organizado de atividades, na forma de um plano, que podem ser utilizadas para projetar a organização e o desdobramento do mesmo. Os projetos de processos podem buscar a produção com custos reduzidos, produtos de alta qualidade, satisfação dos clientes e inovação constante.

Andersen (2006), argumenta que as diversas formas de gerenciar um projeto, podem ser atribuídas pela sua característica, complexidade, setor de atuação, tipo do cliente e demais necessidades específicas. Diante destas necessidades, destaca-se a importância da evolução e descoberta de novas metodologias que possibilitem o gerenciamento deles de uma forma integrada à operação.

\section{Apresentação e Análise de Resultados}

Nesta seção, serão apresentados os dados referentes a publicação, com seus devidos períodos e locais, análise dos autores e de grupo de palavras-chave no tema gestão estratégica de operações de serviço e organização por projeto. Após a organização dos dados, foram construídas redes de relacionamento para um grupo selecionado de autores e das respectivas palavras-chave, constantes nas suas publicações. São calculados também os indicadores de densidade, centralidade, centralização, intermediação e proximidade para as redes construídas.

Para Senge (2005), um grupo de indivíduos interligados entre si por meio de um fluxo de informações na forma de redes gera um círculo causal de fatos. Este grupo de indivíduos tem como característica ser alimentado por informações interligadas entre si, projetando um impacto sobre a próxima variável do sistema. 


\subsection{Análise Descritiva}

De acordo com o referencial teórico desenvolvido para se estudar a gestão estratégica de operações de serviço em empresas que trabalham por projetos, as seguintes áreas ou disciplinas foram definidas: Gestão Estratégica de Operações, Operações de Serviço e Gestão de Projetos. Ao definir como as principais áreas da pesquisa: Operações de Serviço, Gestão Estratégica de Operações e Gestão de Projetos, gerou-se uma lista preliminar de palavras-chave para a pesquisa nas bases de dados.

Na Tabela 3 apresenta-se a lista de palavras-chave utilizadas na busca dos artigos. Essas palavras-chave são definidas de acordo com o tema de pesquisa a ser desenvolvido, conforme a interação dos assuntos e objetivo do trabalho.

Tabela 3 - Lista de palavras-chave utilizadas para busca de artigos

\begin{tabular}{c}
\hline Lista de Palavras-chave \\
Controle Gerencial \\
Desenvolvimento de Estratégias \\
Estratégia Gerencial \\
Gerenciamento de Projetos \\
Gestão de Processos Gerenciais \\
Gestão Estratégica de Operações \\
Operações de Serviço \\
Performance de Serviço \\
Serviço \\
Servitizacion \\
Sistemas de Serviços
\end{tabular}

Fonte: Autores (2010)

Após a identificação do grupo de palavras-chave é realizada a busca de artigos nas bases de dados. O critério de escolha para a pesquisa nas bases de dados foi considerado a periodicidade das atualizações, a quantidade de artigos apresentados, a qualidade das informações e a facilidade de busca de artigos publicados dos principais autores, utilizados como referências dos assuntos para o desenvolvimento do trabalho.

A Tabela 4 apresenta a proporção e quantidade de publicações que cada base de dados contribui para a amostra da pesquisa.

Tabela 4 - Pesquisa em banco de dados

\begin{tabular}{|c|c|c|}
\hline Banco de Dados & Quantidade & Percentual \\
\hline Emerald & 18 & $23 \%$ \\
\hline Science Direct & 17 & $22 \%$ \\
\hline Scielo & 13 & $16 \%$ \\
\hline Wiley InterScience & 10 & $13 \%$ \\
\hline $\begin{array}{l}\text { Outros (Harvard Business } \\
\text { Inderscience, Taylor and Francis) }\end{array}$ & 21 & $26 \%$ \\
\hline Total & 79 & $100 \%$ \\
\hline
\end{tabular}

Fonte: Autores (2010) 
As principais bases de dados para pesquisa de artigos utilizados são: Emerald com 18 publicações, Science Direct com 17 publicações, Scielo com 13 publicações e Wiley InterScience com 10 publicações. Vale ressaltar que alguns livros, artigos e dissertações consultados que não estão disponibilizados nas bases de dados do portal de periódicos da CAPES foram contabilizados na amostra.

Para as 79 referências pesquisadas e identificadas nas bases de dados, encontra-se 66 artigos, 12 livros e 1 dissertação. Nesta amostra de pesquisa composta por 79 unidades (publicações), através do estudo de Redes Sociais, identificou-se que 1.365 autores realizaram citações nesta lista de trabalhos publicados. Para realização das simulações no software UCINET 6.109®, foi utilizado apenas as citações dos 20 autores que mais contribuíram e apresentam citações nos trabalhos pesquisados.

O grupo principal dos 20 autores corresponde a 491 citações das 2.916 citações realizadas pelos 1.365 autores da amostra total de trabalhos publicados. O critério de escolha para estudar a analisar os 20 principais autores, tem como objetivo facilitar a análise do autor e focar apenas nas publicações e citações que mais contribuem para o desenvolvimento do trabalho.

Como estratégia de pesquisa, foi realizado o estudo dos trabalhos publicados nos últimos cinco anos (2004-2009). Com essa abordagem, podem-se obter informações mais recentes e aplicadas atualmente nas pesquisas acadêmicas, contribuindo com informações mais atuais. No Gráfico 1, observa-se os principais períodos de publicação do referencial teórico na amostra de 79 trabalhos pesquisados. Na amostra total das publicações, aproximadamente $43 \%$ dos trabalhos foram publicados entre os anos 2004 a 2009, o restante equivale a publicações desde o ano de 1969 com os autores mais clássicos dos assuntos ao ano de 2003 com a contribuição de diversos autores.

Gráfico 1 - Ano de Publicação Referencial Teórico

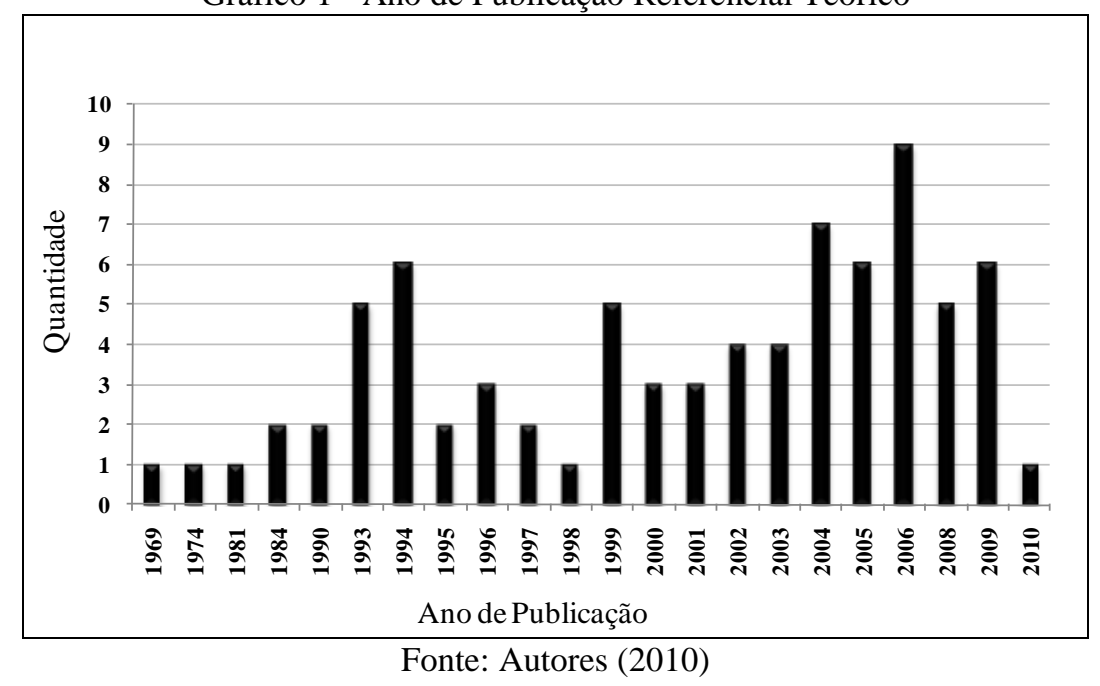


As pesquisas dos periódicos concentram-se em publicações internacionais representando $85 \%$ da amostra de artigos pesquisados e nacionais representam 15\%, conforme a Tabela 5 apresenta.

Tabela 5 - Origem das Publicações

\begin{tabular}{lcc}
\hline \multicolumn{1}{c}{ Origem das Publicações } & Quantidade & Percentual \\
\hline Internacional & 67 & $85 \%$ \\
Nacional & 12 & $15 \%$ \\
Total & 79 & $100 \%$ \\
\hline
\end{tabular}

Fonte: Autores (2010)

A Tabela 6 apresenta a lista de periódicos onde os trabalhos foram publicados.

Tabela 6 - Periódicos Pesquisados

\begin{tabular}{lcc}
\hline \multicolumn{1}{c}{ Periódicos Pesquisados } & Quantidade & Percentual \\
\hline Intern. Journal of Operations \& Production Manag. & 12 & $15 \%$ \\
Journal of Operations Management & 9 & $11 \%$ \\
Harvard Business Review & 8 & $10 \%$ \\
International Journal of Production Economics & 7 & $9 \%$ \\
International Journal of Service Industry Management & 6 & $8 \%$ \\
Outros & 37 & $47 \%$ \\
\hline Total & 79 & $100 \%$ \\
\hline
\end{tabular}

Fonte: Autores (2010)

International Journal of Operations \& Production Management é o periódico que apresenta o maior número de artigos publicados na amostra pesquisada com 12 publicações, seguidos pelos Journal of Operations Management com 9 publicações, Harvard Business Review com 8 publicações, International Journal of Production Economics com 7 publicações e o International Journal of Service Industry Management com 6 publicações. O restante dos periódicos e livros somam 37 publicações.

\subsection{Análise de Redes Sociais - Autores}

A análise sobre os autores também foi submetida ao estudo de redes. Através de uma busca pelo número de citações que cada autor contribui na amostra de 79 unidades de pesquisa, conforme a Tabela 7 apresenta. JOHNSTON, R., WHEELWRIGHT, G. S., HAYES, H., VOSS, C. e CHASE, R. foram os autores que mais obtém citações no grupo de referências pesquisadas. Estes autores fazem citações ou receberam citações sobre assuntos relacionados às áreas de delimitação da pesquisa.

Como critério de decisão para análise da Rede Social, utilizou-se os 20 autores que mais obtiveram citações na amostra de pesquisa. A amostra de 20 indivíduos permite uma melhor interpretação e análise dos dados gerados após a simulação das informações software UCINET 6.109®. Caso o pesquisador utilize uma amostra maior de autores, os gráficos gerados com as 
simulações apresentam um formato mais complexo e uma dimensão maior, dificultando a interpretação dos dados e informações.

Tabela 7 - Principais autores citados - Referencial Teórico

\begin{tabular}{lc}
\hline Autores mais citados & Quantidade \\
\hline Johnston, R. (1987-2005) & 55 \\
Wheelwright, G.S. (1979) & 47 \\
Hayes, H. (1979-2005) & 44 \\
Voss, C. (1986-2007) & 38 \\
Chase, R. (1973-2000) & 32 \\
Platts, K. (1990-2006) & 29 \\
Lovelock, C. (1982-1993) & 25 \\
Bowen, D.(1985-1998) & 24 \\
Skinner, W. (1969-1994) & 24 \\
Neely, A. (1993-2004) & 23 \\
Mills, J. (1986-2003) & 21 \\
Fitzgerald, L. (1990-1993) & 20 \\
Hill, A. (1987-2001) & 17 \\
Biciti, U. (1999-2004) & 16 \\
Silvestro, R. (1991) & 15 \\
Slack, N. (1983-2004) & 14 \\
Porter, M. (1980-1994) & 14 \\
Acur, N. (2000-2004) & 13 \\
Hill, T. (1977-1999) & 10 \\
Schmenner, R. (1977-2003) & 10 \\
\hline Total & 491 \\
\hline Fonte: Autores (2010) &
\end{tabular}

O grupo dos 20 principais autores como apresenta a Tabela 7, corresponde a $17 \%$ do percentual total, com 491 citações, referindo-se ao número total de 2.916 citações. $2 \%$ dos autores são responsáveis por $17 \%$ das citações totais encontradas na amostra da pesquisa.

A citação dos autores em vários artigos ocorre a repetição da mesma citação e do mesmo autor, sendo assim esse número de 2.916 é reduzido para 1.345 autores quando eliminadas as repetições. Essa diminuição é devido a exclusão da repetição, sendo contabilizado apenas o mesmo autor e não as repetições que o mesmo autor apresenta.

Na Figura 4, observa-se a relação dos indivíduos dentro do grupo de autores ou da rede de relacionamentos, conforme resultados das simulações do software UCINET 6.109®, utilizado como ferramenta de apoio para analise e interpretação dos dados. 


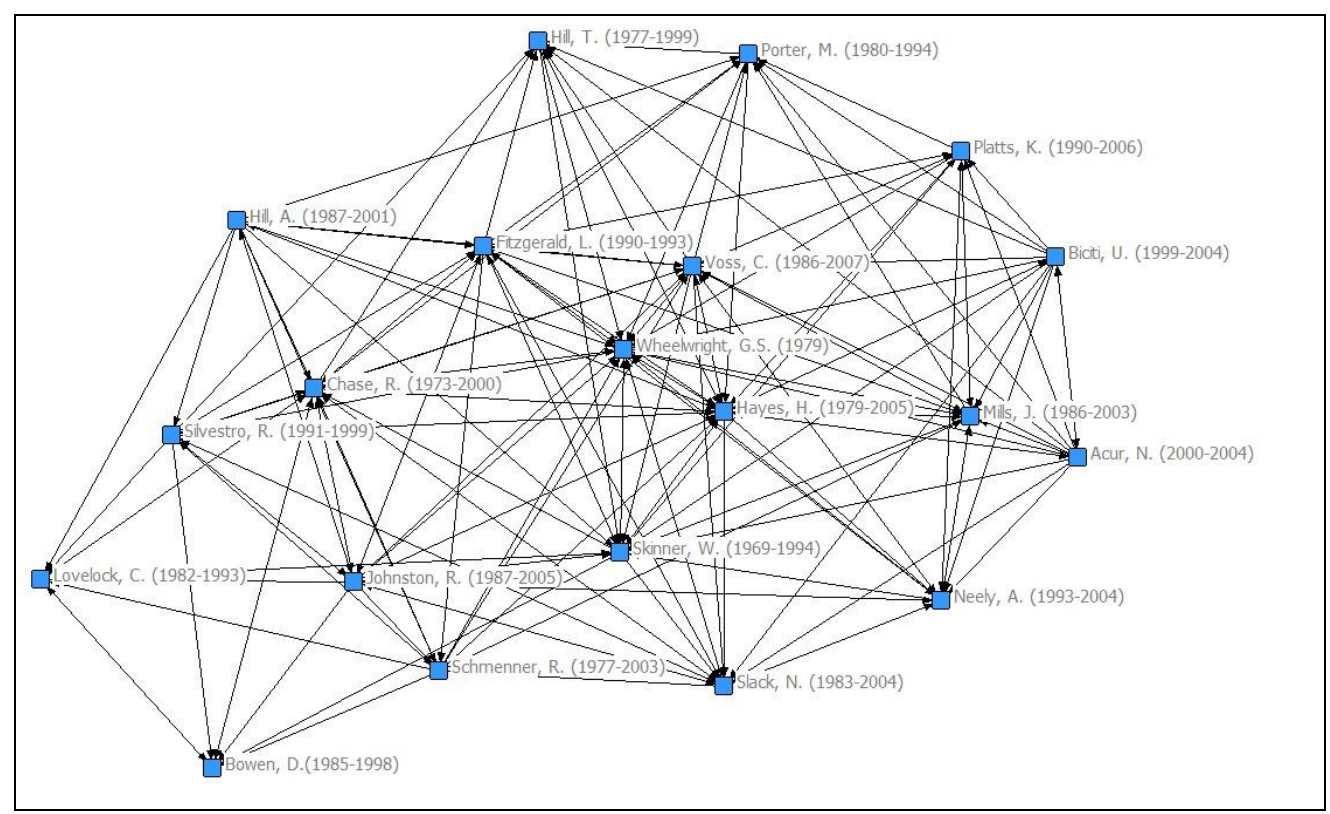

Fonte: Autores (2010)

A Figura 4 observa-se a relação apresentada por cada autor sobre outros indivíduos (autores). Os autores que estão no centro da rede apresentam maiores números de citações, recebendo ou realizando a citação. Pode-se comprovar através de uma comparação com a Tabela 8, sendo os autores que contribuem com o maior número de citações, a posição no centro da rede conforme a Figura 4.

\subsection{Grau de Centralização}

De acordo com Alejandro e Norman (2006), o indicador de centralização é a relação direta que o autor tem sobre outros autores em forma de fluxo bidirecional, sendo a transmissão e recepção das informações. Os autores que estão no centro da rede possuem maior grau de conectividade por apresentarem maiores volumes de citações. JOHNSTON, R. (1987-2005), o autor cujas citações são maiores que demais indivíduos do grupo, sendo apresentado pelo grau de Normalização de Entrada com 20.000 pontos e Normalização Saída com 53.000 pontos conforme a Tabela 8.

Tabela 8 - Principais Autores Indicadores de Centralização

\begin{tabular}{llccc}
\hline \multicolumn{1}{c}{ Autores } & $\begin{array}{c}\text { Grau } \\
\text { Saída }\end{array}$ & $\begin{array}{c}\text { Grau } \\
\text { Entrada }\end{array}$ & $\begin{array}{c}\text { Normalização } \\
\text { Saída }\end{array}$ & $\begin{array}{c}\text { Normalização } \\
\text { Entrada }\end{array}$ \\
\hline Johnston, R. (1987-2005) & 53.000 & 20.000 & 27.895 & 10.526 \\
Fitzgerald, L. (1990-1993) & 36.000 & 19.000 & 18.947 & 10.000 \\
Acur, N. (2000-2004) & 35.000 & 8.000 & 18.421 & 4.211 \\
Silvestro, R. (1991-1999) & 33.000 & 12.000 & 17.368 & 6.316 \\
Chase, R. (1973-2000) & 30.000 & 29.000 & 15.789 & 15.263 \\
Biciti, U. (1999-2004) & 25.000 & 12.000 & 13.158 & 6.316 \\
Hayes, H. (1979-2005) & 23.000 & 20.526 & 12.105 & 39.000
\end{tabular}


Hill, A. (1987-2001)

Mills, J. (1986-2003)

Platts, K. (1990-2006)

Voss, C. (1986-2007)

Skinner, W. (1969-1994)

Hill, T. (1977-1999)

Schmenner, R. (1977-2003)

Neely, A. (1993-2004)

Porter, M. (1980-1994)

Lovelock, C. (1982-1993)

Wheelwright, G.S. (1979)

Slack, N. (1983-2004)

Bowen, D.(1985-1998)

Fonte: Autores (2010)

\begin{tabular}{cccc}
22.000 & 2.000 & 11.579 & 1.053 \\
17.000 & 17.000 & 8.947 & 8.947 \\
16.000 & 24.000 & 8.421 & 12.632 \\
14.000 & 32.000 & 7.368 & 16.842 \\
13.000 & 22.000 & 6.842 & 11.579 \\
11.000 & 12.000 & 5.789 & 6.316 \\
11.000 & 8.000 & 5.789 & 4.211 \\
10.000 & 22.000 & 5.263 & 11.579 \\
8.000 & 12.000 & 4.211 & 6.316 \\
7.000 & 15.000 & 3.684 & 7.895 \\
7.000 & 42.000 & 3.684 & 22.105 \\
6.000 & 16.000 & 3.158 & 8.421 \\
4.000 & 18.000 & 2.105 & 9.474 \\
\hline
\end{tabular}

Realizando-se uma comparação com a Figura 4 e com a Tabela 8, observa-se que JOHNSTON, R. (1987-2005), está no centro da Rede Social e apresenta maiores índices de pontuação em realização aos graus de Normalização dos demais indivíduos. Esta análise pode ser realizada para os outros autores, observando-se a posição na Rede Social e o nível de pontuação obtida pelos índices de normalização.

Na Tabela 9 apresentam-se a descrição dos indicadores estatísticos do software UCINET $6.109 \circledR$ gerado para as simulações do grau de conectividade.

Tabela 9 - Definição dos Indicadores Gerais da Rede

\begin{tabular}{cc}
\hline Indicadores & Descrição dos Indicadores Gerais da Rede \\
\hline Mean & Media: é a relação média de todos os indicadores \\
Std Dev & Desvio Padrão: variação da média geral \\
Sum & Soma: é a soma de todas as relações da rede \\
Variance & Variação media da soma de todas as relações da rede \\
SSQ & Distância média de um indivíduo e outro \\
MCSSQ & Média geral do indivíduo, capacidade de relação na rede \\
Euc Norm & Média geral do índice de normalização \\
Minimum & Quantidade mínima de interações \\
Maximum & Quantidade máxima de interações \\
\hline
\end{tabular}

Fonte: Autores (2010)

As estatísticas são utilizadas para demonstrar a média percentual de todos os indicadores da rede, como média, desvio padrão e graus mínimos e máximos de interações entre os indivíduos, conforme a simulação do grupo de autores, na Tabela 10.

Tabela 10 - Estatística Geral - Indicadores de Centralização Autores

\begin{tabular}{ccccc}
\hline Média geral & $\begin{array}{c}\text { Grau } \\
\text { Saída }\end{array}$ & $\begin{array}{c}\text { Grau } \\
\text { Entrada }\end{array}$ & $\begin{array}{c}\text { Normalização } \\
\text { Saída }\end{array}$ & $\begin{array}{c}\text { Normalização } \\
\text { Entrada }\end{array}$ \\
\hline Mean & 19.050 & 19.050 & 10.026 & 10.026 \\
Std Dev & 12.580 & 9.997 & 6.621 & 5.262 \\
Sum & 381.000 & 381.000 & 200.526 & 200.526 \\
Variance & 158.247 & 99.948 & 43.836 & 27.686 \\
SSQ & 10423.000 & 9257.000 & 2887.258 & 2564.266 \\
MCSSQ & 3164.950 & 1998.950 & 876.717 & 553.726 \\
Euc Norm & 102.093 & 96.213 & 53.733 & 50.639 \\
Minimum & 4.000 & 2.000 & 2.105 & 1.053 \\
Maximum & 53.000 & 42.000 & 27.895 & 22.105 \\
\hline
\end{tabular}

Fonte: Autores (2010) 
Os indicadores estatísticos são indicadores que auxiliam e aprofundam a análise sobre os resultados das simulações, possibilitando ao pesquisador outra fonte de informações para o entendimento da Rede Social.

\subsection{Grau de Densidade}

Outro tipo de simulação que o software UCINET 6.109® apresenta são os indicadores de Densidade. A Densidade é o grau de conectividade que o indivíduo apresenta dentro da rede social, sendo a média geral das interações de todos os indivíduos do grupo (ALEJANDRO e NORMAN, 2006).

Este índice pode ser calculado pela fórmula $R P=N T N \times(N T N-1)$ e $D=R E / R P \times 100$ onde: $R P$ - relações possíveis, $N T N$ - número total de nós da rede, $R E$ - relações existentes.

O resultado do cálculo de Densidade da Rede Social para o grupo de autores é dado por:

$$
\begin{array}{lrl}
R P & =20 \text { (nós da rede) } \times 20 \text { (nós da rede) }-1 & D=\underline{491} \\
R P=20 \times 19=380 & 380 \times 100=\mathbf{1 2 \%}
\end{array}
$$

Para o grau de densidade da Rede Social dos autores conforme resultados da simulação é de $12 \%$, sendo o nível médio de conectividade entre todos os indivíduos. Para essa simulação, encontra-se 380 possíveis relações que um autor pode apresentar dentro do grupo e 491 é o numero total de relações dentro da rede, sendo a quantidade de citações realizadas e recebidas por todos os autores do grupo.

Quanto maior é o índice de densidade (percentual), maior é a conectividade que o autor (indivíduo) apresenta um ao outro dentro do mesmo grupo, sendo uma média geral entre todos os membros da Rede Social.

\subsection{Grau de Intermediação}

Segundo Alejandro e Norman (2006), a simulação para análise do grau de intermediação é a possibilidade que um indivíduo tem para intermediar um fluxo de informação, sendo a possibilidade que um autor (indivíduo) pode realizar os caminhos geodésicos. Pode ser a ligação entre um autor e outro grupo de autores.

A Tabela 11 apresenta informações referentes aos indicadores de intermediação da rede social dos autores. 
Tabela 11 - Indicadores de Intermediação

\begin{tabular}{lcc}
\hline \multicolumn{1}{c}{ Autores } & Betweenness & nBetweenness \\
\hline Hayes, H. (1979-2005) & 55.154 & 16.127 \\
Chase, R. (1973-2000) & 49.071 & 14.348 \\
Skinner, W. (1969-1994) & 35.745 & 10.452 \\
Wheelwright, G.S. (1979) & 25.813 & 7.548 \\
Slack, N. (1983-2004) & 23.088 & 6.751 \\
Fitzgerald, L. (1990-1993) & 22.281 & 6.515 \\
Voss, C. (1986-2007) & 15.136 & 4.426 \\
Hill, A. (1987-2001) & 9.619 & 2.813 \\
Johnston, R. (1987-2005) & 8.086 & 2.364 \\
Schmenner, R. (1977-2003) & 4.727 & 1.382 \\
Porter, M. (1980-1994) & 4.542 & 1.328 \\
Silvestro, R. (1991-1999) & 3.537 & 1.034 \\
Mills, J. (1986-2003) & 2.918 & 0.853 \\
Neely, A. (1993-2004) & 2.698 & 0.789 \\
Bowen, D.(1985-1998) & 2.185 & 0.639 \\
Lovelock, C. (1982-1993) & 1.652 & 0.483 \\
Platts, K. (1990-2006) & 1.480 & 0.433 \\
Hill, T. (1977-1999) & 0.619 & 0.181 \\
Biciti, U. (1999-2004) & 0.325 & 0.095 \\
Acur, N. (2000-2004) & 0.325 & 0.095 \\
\hline Fonte: Autores (2010) & &
\end{tabular}

Fonte: Autores (2010)

HAYES, H. (1979-2005), CHASE, R. (1973-2000) e SKINNER, W. (1969-1994) são os autores com maiores níveis de intermediação, característica que pode ser comprovada com a posição por eles ocupadas na rede social, posicionados no centro do gráfico.

A intermediação é a característica que o autor intermedia o fluxo de informações entre outros autores, sendo responsável por interagir e comunicar com outros indivíduos do grupo.

\subsection{Grau de Proximidade}

O grau de proximidade é a capacidade que um indivíduo tem para se relacionar com todos ou o maior número de indivíduos da rede. É a distância que um autor está distante de outro grupo de autores, sendo possível calcular este espaço.

A Tabela 12 apresenta os indicadores de proximidade existente no grupo de autores (ALEJANDRO e NORMAN, 2006).

Tabela 12 - Indicadores de Proximidade

\begin{tabular}{lcccc}
\hline \multicolumn{1}{c}{ Autores } & inFarness & outFarness & inCloseness & outCloseness \\
\hline Wheelwright, G.S. (1979) & 21.000 & 37.000 & 90.476 & 51.351 \\
Hayes, H. (1979-2005) & 24.000 & 29.000 & 79.167 & 65.517 \\
Skinner, W. (1969-1994) & 25.000 & 32.000 & 76.000 & 59.375 \\
Voss, C. (1986-2007) & 26.000 & 30.000 & 73.077 & 63.333 \\
Chase, R. (1973-2000) & 28.000 & 27.000 & 67.857 & 70.370 \\
Slack, N. (1983-2004) & 29.000 & 35.000 & 65.517 & 54.286 \\
Hill, T. (1977-1999) & 30.000 & 40.000 & 63.333 & 47.500 \\
Neely, A. (1993-2004) & 31.000 & 39.000 & 61.290 & 48.718 \\
Mills, J. (1986-2003) & 32.000 & 35.000 & 59.375 & 54.286
\end{tabular}




\begin{tabular}{lllll} 
Fitzgerald, L. (1990-1993) & 32.000 & 25.000 & 59.375 & 76.000 \\
Bowen, D.(1985-1998) & 32.000 & 43.000 & 59.375 & 44.186 \\
Platts, K. (1990-2006) & 33.000 & 35.000 & 57.576 & 54.286 \\
Porter, M. (1980-1994) & 35.000 & 34.000 & 54.286 & 55.882 \\
Schmenner, R. (1977-2003) & 36.000 & 31.000 & 52.778 & 61.290 \\
Johnston, R. (1987-2005) & 37.000 & 25.000 & 51.351 & 76.000 \\
Hill, A. (1987-2001) & 38.000 & 27.000 & 50.000 & 70.370 \\
Silvestro, R. (1991-1999) & 38.000 & 27.000 & 50.000 & 45.238 \\
Lovelock, C. (1982-1993) & 40.000 & 42.000 & 47.500 & 67.857 \\
Biciti, U. (1999-2004) & 41.000 & 28.000 & 46.341 & 67.857 \\
Acur, N. (2000-2004) & 41.000 & 28.000 & 46.341 & \\
\hline
\end{tabular}

Fonte: Autores (2010)

A distância é a soma dos intervalos entre os autores, quando mais alto o valor de proximidade, maior a capacidade que o indivíduo tem para se conectar e interagir com outros indivíduos. O grau de proximidade pode ser expresso pelo indicador de entrada (inFarness/inCloseness) e indicador de saída (outFarness/outCloseness).

\subsection{Análise de Redes - Palavras-Chave}

Busca-se com o estudo de redes observarem quais palavras tem uma relação direta ou indireta na delimitação da pesquisa. As palavras-chave que apresentaram maiores contribuições nas pesquisas foram: 'serviço' com 40 citações, 'controle gerencial' com 27 e 'competitividade' com 20 citações. Esse grupo de palavras foi extraído dos artigos utilizados para o desenvolvimento do referencial teórico para o desenvolvimento do artigo. A Tabela 13 apresenta as principais palavraschave pesquisadas.

Tabela 13 - Principais Palavras-chave pesquisadas Referencial Teórico

\begin{tabular}{lcc}
\hline \multicolumn{1}{c}{ Palavras-chave } & Quantidade & Percentual \\
\hline Serviço & 40 & $23 \%$ \\
Controle Gerencial & 27 & $15 \%$ \\
Competitividade & 20 & $11 \%$ \\
Gestão de Operações & 18 & $10 \%$ \\
Gestão Estratégica & 18 & $10 \%$ \\
Metodologia & 17 & $10 \%$ \\
Processo & 13 & $7 \%$ \\
Projetos & 11 & $6 \%$ \\
Desenvolvimento & 8 & $5 \%$ \\
Análise & 5 & $3 \%$ \\
\hline
\end{tabular}

Fonte: Autores (2010)

Foi necessário realizar uma subdivisão do grupo principal das palavras-chave, sendo que a mesma facilita a identificação de outros trabalhos que apresentam palavras-chave diferentes do principal grupo, porém fazem parte da mesma área ou assunto de pesquisa. Dessa forma é possível identificar mais trabalhos relacionados ao mesmo assunto da pesquisa, como resultado a amostra apresentará maior número de informações. Como exemplo, palavras-chave de outros idiomas (inglês) são traduzidas para o português, apresentando o mesmo sentido e a área da pesquisa. 
Ao realizar a simulação dos dados no software UCINET 6.109®, observa-se uma relação direta entre as palavras mais citadas conforme a Figura 4. Serviço, controle gerencial, competitividade, gestão estratégica e gestão de operações são palavras-chave que apresentam maior grau de centralidade dentro da rede social para este grupo.

O grau de centralidade é a característica apresentada pelos indivíduos quando estes estão relacionados diretamente com outros indivíduos. Aqueles que estão no centro da rede apresentam maior grau de conectividade em relação aos demais, conforme a Figura 5 demonstra.

Figura 5 - Análise de Redes Sociais Palavras-chave

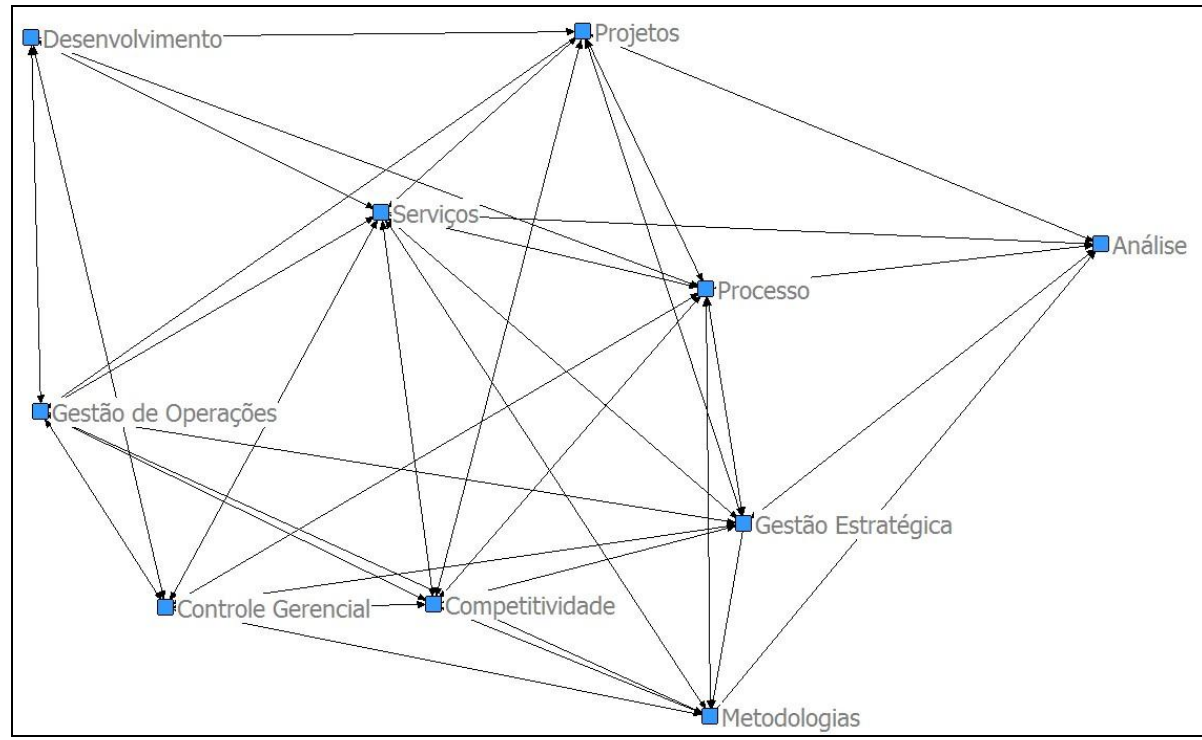

Fonte: Autores (2010)

Pode-se dizer que a palavra-chave que maior apresenta citações recebidas é a palavra 'serviço', por apresentar o maior grau de Normalização de Entradas, sendo 32.222 pontos conforme a Tabela 14 apresenta.

A Normalização de Saídas é a característica que o indivíduo realiza as citações, nesta situação a palavra Serviço também é responsável pela realização de citações para outros indivíduos com a pontuação de 43.333. Essa pontuação quanto maior o seu nível, maior é a capacidade que o autor (indivíduo) tem para realizar citações de outros autores, interagindo e trocando informações dentro da Rede Social.

\subsection{Análise de Cálculo de Indicadores - Palavras-Chave}

O software UCINET $6.109 \circledR$ tem como ferramenta de apoio a simulação, a geração de indicadores estatísticos para auxilio e maior aprofundamento das análises dos cenários da Rede Social. De acordo com os indicadores estatísticos, conforme o menor grau de Normalização de Entradas e Saídas, menor é a relação e citações que o indivíduo apresenta no grupo. 
O grau de centralização da palavra 'serviço' pode-se ser comparada com a posição que este indivíduo está localizado na parte central da rede, conforme a Figura 5 e com os resultados da Tabela 14, sucessivamente para as outras palavras e indivíduos.

Tabela 14 - Principais Palavras-chave pesquisadas Indicadores de Centralização

\begin{tabular}{lcccc}
\hline \multicolumn{1}{c}{ Palavras-chave } & $\begin{array}{c}\text { Grau } \\
\text { Saída }\end{array}$ & $\begin{array}{c}\text { Grau } \\
\text { Entrada }\end{array}$ & $\begin{array}{c}\text { Normalização } \\
\text { Saída }\end{array}$ & $\begin{array}{c}\text { Normalização } \\
\text { Entrada }\end{array}$ \\
\hline Serviço & 39.000 & 29.000 & 43.333 & 32.222 \\
Controle Gerencial & 24.000 & 19.000 & 26.667 & 21.111 \\
Competitividade & 20.000 & 19.000 & 22.222 & 21.111 \\
Gestão Estratégica & 17.000 & 16.000 & 18.889 & 17.778 \\
Gestão de Operações & 15.000 & 25.000 & 16.667 & 27.778 \\
Metodologia & 14.000 & 14.000 & 15.556 & 15.556 \\
Projetos & 11.000 & 7.000 & 12.222 & 7.778 \\
Processo & 11.000 & 23.000 & 12.222 & 25.556 \\
Desenvolvimento & 8.000 & 5.000 & 8.889 & 5.556 \\
Análise & 3.000 & 5.000 & 3.333 & 5.556 \\
\hline
\end{tabular}

Fonte: Autores (2010)

De acordo com a amostra de 79 trabalhos publicados, utilizados para realizar as simulações e estudo das Redes Sociais, a palavra-chave 'Serviço' apresenta maior relevância para a pesquisa, sendo um dos assuntos a ser desenvolvido no estudo, visando o entendimento sobre a interação de autores e palavras-chave, das disciplinas de Gestão Estratégica de Operações, Operações de Serviço e Organização por Projetos, gerando uma análise através da metodologia de Redes Sociais.

A Figura 5 representa a aproximação que a palavra-chave 'Serviço' esta com outros assuntos, como Gestão Estratégica, Gestão de Operações e Projetos. Os indicadores estatísticos 43.333 e 32.222 conforme a tabela 14 apresenta, podem comprovar através dos índices de entradas e saídas, sendo o nível de interação que a palavra-chave 'Serviço' tem com outros assuntos estudados.

Em uma próxima etapa, outro artigo apresentará a interação dessas palavras-chave para o desenvolvimento de estudos de Casos múltiplos e a aplicação de um modelo de Gestão Estratégica de Operações em organizações que trabalham por sistemas de Serviço e Projetos. Esse modelo de Gestão Estratégica de Operações servirá como uma ferramenta de apoio para o desdobramento de processos ligados a essa área, formalizando as etapas para desenvolvimento, implementação e controle da Gestão Estratégica.

\section{Quadro Síntese}

Através de um quadro descritivo, nesta seção serão apresentadas as principais contribuições alcançadas nesse trabalho resumindo as informações mais relevantes da pesquisa. 
Quadro 1 - Definição dos Indicadores Gerais da Rede

\begin{tabular}{|c|c|c|}
\hline \multicolumn{2}{|r|}{ Resultados } & Contribuição \\
\hline \multirow{5}{*}{$\begin{array}{l}\text { Análise de } \\
\text { Redes } \\
\text { Sociais: } \\
\text { Autores }\end{array}$} & $\begin{array}{l}\text { 1. Quantidade de } \\
\text { Citações }\end{array}$ & $\begin{array}{l}\text { Autores que apresentaram maior número de citações na amostra } \\
\text { pesquisada: } \\
\text { Johnston, R. (1987-2005), Wheelwright, G.S. (1979), Hayes, H. (1979- } \\
\text { 2005), Voss, C. (1986-2007) e Chase, R. (1973-2000). }\end{array}$ \\
\hline & $\begin{array}{l}\text { 2. Indicadores de } \\
\text { Centralização }\end{array}$ & $\begin{array}{l}\text { Autores que apresentam maior nível de recebimento e realização de troca } \\
\text { de informações: } \\
\text { Johnston, R. (1987-2005), Fitzgerald, L. (1990-1993), Acur, N. (2000- } \\
\text { 2004), Silvestro, R. (1991-1999) e Chase, R. (1973-2000). }\end{array}$ \\
\hline & $\begin{array}{l}\text { 3. Indicadores de } \\
\text { Densidade }\end{array}$ & $\begin{array}{l}\text { A Densidade é o grau de conectividade que o indivíduo apresenta dentro } \\
\text { da rede social, sendo a média geral das interações de todos os indivíduos } \\
\text { do grupo }-12 \%\end{array}$ \\
\hline & $\begin{array}{l}\text { 4.Indicadores de } \\
\text { Intermediação }\end{array}$ & $\begin{array}{l}\text { Possibilidade que um indivíduo tem para intermediar um fluxo de } \\
\text { informação. } \\
\text { Hayes, H. (1979-2005), Chase, R. (1973-2000), Skinner, W. (1969-1994), } \\
\text { Wheelwright, G.S. (1979) e Slack, N. (1983-2004). }\end{array}$ \\
\hline & $\begin{array}{l}\text { 5.Indicadores de } \\
\text { Proximidade }\end{array}$ & $\begin{array}{l}\text { Capacidade que os autores tem para se relacionar com outros autores. } \\
\text { Wheelwright, G.S. (1979), Hayes, H. (1979-2005), Skinner, W. (1969- } \\
\text { 1994), Voss, C. (1986-2007) e Chase, R. (1973-2000). }\end{array}$ \\
\hline \multirow{2}{*}{$\begin{array}{l}\text { Análise de } \\
\text { Redes } \\
\text { Sociais: } \\
\text { Palavras } \\
\text { chave }\end{array}$} & $\begin{array}{l}\text { 1.Quantidade de } \\
\text { Palavras-chave }\end{array}$ & $\begin{array}{l}\text { Principais Palavras-Chave pesquisadas: } \\
\text { Serviço, Controle Gerencial, Competitividade, Gestão de Operações e } \\
\text { Gestão Estratégica. }\end{array}$ \\
\hline & $\begin{array}{l}\text { 2. Indicadores de } \\
\text { Centralização }\end{array}$ & $\begin{array}{l}\text { Palavras-Chave que apresentam maior nível de recebimento e realização } \\
\text { de troca de informações: } \\
\text { Serviço, Controle Gerencial, Competitividade e Gestão Estratégica. }\end{array}$ \\
\hline
\end{tabular}

Fonte: Autores (2010)

O grupo principal composto pelos autores, que mais apresentaram citações na amostra pesquisada é formado por Johnston, R., Wheelwright, G.S., Hayes, H., Voss, C. e Chase, R. Conforme simulações e visualização do gráfico da Rede Social, esses autores representam maiores pontuações conforme os indicadores estatísticos do software UCINET 6.109®. Pode-se observar que esse grupo, apresenta maior relevância nas citações das amostras de pesquisa, sendo assim a busca por artigos e trabalhos publicados para o desenvolvimento da pesquisa, será direcionada para esse grupo de autores.

As revistas mais utilizadas para a busca das publicações são: International Journal of Operations \& Production Management, Journal of Operations Management, Harvard Business Review, International Journal of Production Economics e International Journal of Service Industry Management. Essas revistas formam o grupo mais relevante da amostra pesquisada, sendo responsável pelo maior número de publicações encontradas nas referências pesquisadas. 


\section{Agenda de Pesquisa}

Nesta seção do trabalho apresentam-se algumas discussões sobre novos temas de pesquisa, havendo continuidade em uma etapa futura, visando o desenvolvimento para outros assuntos e direcionamento para investigação de outras pesquisas.

a) Tema 01 - Quando o processo de desdobramento de estratégias de negócios segue um modelo conceitual e sistematizado, é possível haver mais controle e acompanhamento dos processos, como resultado a organização pode apresentar mais competitividade sobre o mercado e seus concorrentes (ACUR e BITITCI, 2004).

De acordo com a citação, argumenta-se para uma abordagem ligada ao desenvolvimento de modelos para desdobramento de Gestão Estratégica de Operações, visando:

- QP1: Qual o impacto no resultado ao desdobrar uma estratégia, utilizando um modelo sistematizado em forma de processos?

- QP2: Como é possível medir o resultado atingido com a utilização de uma metodologia para desdobramento de estratégias?

- QP3: É possível integrar informações do mercado e informações internas da organização através de um modelo conceitual que seja flexível e adaptável para a necessidade específica de cada organização?

b) Tema 02 - Para Killen e Hunt (2010), a diminuição do ciclo de vida dos produtos e o avanço repentino das evoluções tecnológicas, indicam a necessidade de métodos e técnicas para alinhar estratégias e processos de decisão ligados ao setor de serviços. O desenvolvimento de processos ligados a controle, qualidade e inovação de serviços, traz novas técnicas de desenvolvimento de projetos de produtos ligados a serviços. (SIDHARTHA e CEM, 2006).

Conforme a necessidade de contribuição para desenvolvimento de estudos e metodologias para Operações de Serviço, argumenta-se:

- QP1: Qual a contribuição para o desenvolvimento de um modelo de Gestão Estratégica de Operações para Operações de Serviço, levando em consideração a necessidade de novas metodologias e ferramentas?

- QP2: O modelo desenvolvido de Gestão Estratégica de Operações atende as necessidades de Operações de Serviço?

Um dos pontos fundamentais para o avanço em novas pesquisas é o desenvolvimento de um modelo conceitual para organizações que atuem em sistemas de Projetos, nas Operações de Serviço, 
desdobrando suas estratégias com a utilização de um modelo que permite controlar e ajustar os processos através da sistematização da Gestão Estratégica de Operações.

\section{Considerações Finais}

A análise de Redes Sociais apresenta a interação entre indivíduos e classes sociais de uma comunidade ou um grupo, cujos interesses são comuns. Essa relação dos indivíduos interligados através de objetivos em comum representa uma ação e um impacto sobre outro indivíduo formando um círculo de fatos, troca de idéias e de informações, constituindo a formação de uma Rede Social.

Através das redes ou gráficos, foi possível de forma objetiva, identificar as características que cada indivíduo possui dentro da rede de relacionamentos. Neste caso, para o grupo de autores foi identificado qual indivíduo apresenta maior influência na amostra de artigos e livros utilizados para o desenvolvimento da pesquisa. Esta influência, como exemplo, pode ser o número de citações que o autor recebeu e a quantidade de trabalhos publicados em um período de tempo.

Para a análise de palavras-chave é possível identificar a relação que uma palavra tem com determinado assunto, quando realizada a busca dos artigos nas bases de dados e portais de pesquisa. O resultado da análise orienta o pesquisador para o direcionamento da coleta das informações para a pesquisa nas bases de dados. A busca direcionada de artigos e trabalhos publicados trará ao pesquisador maior qualidade dos dados, pelo entendimento do estado da arte do assunto pesquisado. O estado da arte pode ser a definição atribuída para a importância que o tema da pesquisa encontrase no momento, tendo informações sobre as áreas de publicações, como quantidade, autores que atuam em projetos de pesquisas, assuntos estudados e evolução das pesquisas nessa área.

A contribuição para a Gestão Estratégica de Operações oferece uma visão sobre o levantamento de publicações que um grupo de autores apresenta para essa área. É possível analisar a interação que esses autores obtêm com outros autores (rede social), identificando a quantidade de publicações e evoluções das pesquisas que esse mesmo grupo apresenta para a sociedade acadêmica.

Pelo fato de existir poucos trabalhos publicados sobre análise de redes com utilização do software UCINET 6.109® ou outra ferramenta equivalente, o acesso para esse tipo de conteúdo é limitado.

Este trabalho terá continuidade em outra seqüência, sendo possível com este tipo de análise a realização de estudos para outros campos de pesquisa, como referencial teórico para análise de estudos de Caso. A utilização dessa metodologia pode ser aplicada em estudos de Caso pelo fato da ferramenta (software UCINET 6.109®) possibilitar a simulação para outros campos de pesquisa, sendo possível realizar simulações de dados quantitativos e qualitativos. 
A utilização de outras ferramentas e metodologias para análise de referencial teórico contribui para a evolução e diferenciação de pesquisas acadêmicas sobre essa disciplina. Esse tipo de análise tem como característica de forma simples a visualização da Rede Social, através dos gráficos e figuras, sendo também auxiliada com indicadores estatísticos para aprofundamento da interpretação dos dados, oferecendo ao pesquisador mais um forma de interpretação dos dados.

\begin{abstract}
Operations strategic management has as objective to contribute for sustaining the competitive positioning of companies in their served markets, taking care of customers' needs and generating competitive advantages in relation to their competitors. Following markets' evolution, some sectors initiate a transformation process, as an example, the occurred modifications in services sector where, in recent years, its participation in countries' economy has grown. This article has as an objective of assessing the service operations strategic management scientific production, particularly related to project based enterprise studies. The research strategy is based on the analysis of social nets that allows mapping relationships between authors, keywords and journals. The analysis results in a comprehension of the present stage of research studies in the selected theme and it shows opportunities for future studies. The study objectively identified through graphs and tables which are groups of individuals, as authors and keywords, most influenced the studied sample, guiding the researcher in constructing a better understanding of the state of the art of the studied subject, as well as pointing the main themes and issues for future research. This work contributes for developing new applications related to the study of social nets, being possible through the analysis, to identify the quality of this information for future research development.
\end{abstract}

Keywords: operations strategic management, operations services, project organization, social networks.

\title{
Referências
}

ABREU, J. C. A.; PINHO, J. A. G. Movimentos Sociais e a Reforma Política: Uma análise baseada em Redes Sociais. SEGET - Simpósio de Excelência em Gestão e Tecnologia, 2008.

ACUR, N.; BITITCI, U. A balanced approach to strategy process. International Journal of Operations and Production Management, v.24, n.4, p.388-408, 2004.

cross ref

ALEJANDRO, V. A. O.; NORMAN, A. G. Manual introdutório das Redes Sociais. Centro de Capacitacion e Evaluacion, para El Desarrollo Rural, 2006. Disponível em: <http://revistaredes.rediris.es/webredes/talleres/Manual_ARS.pdf. > Acesso em 2 mar.2010, 16:30:00.

ANDERSEN, E. S. Toward a Project Management Theory for Renewal Projects. Project Management Journal, v.37, n.4, p.15-30, 2006

ARAUJO, L.; SPRINGER, M. Service, products, and institutional structure of production. Industrial Marketing Management. v.35, n.7, p.797-805, 2006

BORGATTI, S.P.; EVERETT, M.G.; FREEMAN, L.C. Ucinet for Windows: Software for Social Network Analysis. Harvard, MA: Analytic Technologies, 2002. 
BOWEN, D.E.; CHASE, R.B.; CUMMINGS, T.G. and et al. Service Management Effectiveness, Jossey-Bass, San Francisco, CA, 1990.

BRAX, S.A. A manufacturing becoming service provider - challenger and paradox. Management Service Quality, v.15, n.2, p.142-155, 2005.

cross ref

CAMPBELL, A.; LUCHS, S.K. Core Competency-Based Strateg. International Thomson Business Press, London, 1997.

CAPES. Coordenação de Aperfeiçoamento de Pessoal de Nível Superior Disponível em: <.http://www.capes.gov.br/ > Acesso em 2 mar.2010.

CAPRA, F. A Teia da Vida: uma nova compreensão cientifica dos sistemas vivos. 8. ed. São Paulo: Cultrix, 2003.

GIANESI, I. G. N.; CORREA, H. L. Administração estratégica de serviços. Operações para a satisfação do cliente. São Paulo: Atlas, 1994.

GYAMPAH, A. K.; MEREDITH, J.R. The operations management research agenda: an update. Journal of Operations Management, v.8, p.250-262, 1989.

cross ref

HAYES, H.R.; PISANO, P.G. Beyond world-class the new manufacturing strategy. Harvard Business Review, v.72, n.10, January-February, p.77-86, 1994.

HAYES, H.R.; UPTON, M.D. Operations based strategy. California Management Review, v.40, n.4, p.8-25, 1998.

HAYES, H.R.; WHEELWRIGHT, G. S. Restoring Our Competitive Edge. Wiley: New York, 1984.

HILl, A. V.; COLliER, D. A.; FROEHLE, C. M; GOODALE, J. C.; METTERS, R. D.; VERMA, R. Research opportunities in service process design. Journal of Operations Management, v.20, p.189-202, 2002.

\section{cross $r$ ref}

HILL, T.J. Manufacturing Strategy. ed 2. Macmillan, Basingstoke, 1993.

KILLEN, C. P.; HUNT, R. A. Dynamic capability through project portfolio management in service and manufacturing industries. International Journal of Managing Projects in Business, v.3, p.157-169, 2010.

cross ref

KOTLER, P. Administração de Marketing: análise, planejamento, implementação e controle. 5. ed. São Paulo: Atlas, 1998.

KOTler, P.; ARMStrong, G. Principles of Marketing. Eighth Edition, Prentice Hall, Englewood Cliffs, New Jersey, 1999.

MACHUCA, J. A.; GONZALEZ, M. M.; ESCOBAR, V.G. Service Operations Management research. Journal of Operations Management, v.25, p.585-603, 2006.

cross ref

MAYRING P. Qualitative Inhaltanalyse - Grundlagen und Techniken. Qualitative content analysis. 8th ed. Weinheim, Germany: Beltz Verlag; 2003.

MILLER, J.G.; GRAHAM, M.B.; FRELAND, J.R.; HOTTENSTEIN, M.; MAISTER, D.M.; MEREDITH, J.; SCHMENNER, R.W. Production/ Operations Management: agenda for the 80s. Decision Science, v.12, p.547-571, 1981.

\section{cross ref}

NEELY, A.D. The Servitizations of Manufacturing. An Analyses of Global Trends. In: POMS, College of Service Operations and EurOMa Conference 2007. Londres: London Business School, 2007. 
NEELY, A.D.; JARRAR, Y. Extracting value from data - the performance planning value chain. Business Process Management Journal, v.10, n.5, p.506-509, 2004.

\section{cross ${ }^{\text {ref }}$}

PILKINGTON, A.; FITZGERALD, R. Operations management themes, concepts and relationships: a forward retrospective of IJOPM. International Journal of Operations \& Production Management, v.26, n.11, p.1255-1275, 2006.

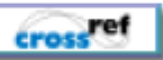

PILKINGTON, A.; LISTON-HEYES, C. Is production and operations management a discipline? A citation/co-citation study', International Journal of Operations \& Production Management, v.19, n.1, p.7-20, 1999.

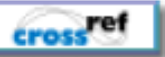

PILKINGTON, A.; MEREDITH, J. The evolution of the intellectual structure of operations management - 1980-2006: a citation/co-citationanalysis. Journal of Operations Management, v. 27, p.185-202, jun. 2009.

\section{cross ref}

PLATTS, K.W.; MILLS, J.F.; GREGORY, M.J. Evaluating strategy formulation processes. International Journal of Production Economics, v. 46, p.233-40, 1996.

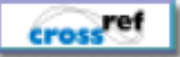

PMBOK. A Guide to Project Management Body of Knowledge - Guide 2004. Edition Project Management Institute - PMI.

REN, G.; GREGORY, M. Servitization in manufacturing companies: a conceptualization, critical review and research agenda. In: Annual Frontiers in Service Conference, 16. San Francisco, CA, USA, 2007.

ROSEN, D.L. The measurement and modeling of quality in service organizations. Unpublished PhD Dissertation, University of South Carolina, 1990.

SCHMENNER, R. W. Administração de Operações em Serviços. São Paulo: Futura, 1986.

SENGE, P. M. A quinta disciplina: a arte e pratica da organização de aprendizagem. ed.19. São Paulo: Best Seler, 2005.

SEURING, S.; MULLER, M. From a literature review to a conceptual framework for sustainable supply chain management. Journal of Cleaner Production, v.16, p.1699-1710, 2008.

cross ref

SHENHAR, A.; DVIR, D. Reinventing Project Management: The Diamond to Successful Growth and Innovation. Boston: Harvard Business School Press, 2007.

SIDHARTHA, R.; CEM C. Designing service processes: a design factor based process model. International Journal Services Technology and Management, v.7, n.1, p.85-107, 2006.

SILVESTRO, R. Positioning services along the volume-variety diagonal. The contingencies of service design, control and improvement. University of Warwick, Coventry, UK. 1999.

SKINNER, W. Manufacturing the missing link in corporate strategy. Harvard Business Review, v.47, n.3, May-June, p.136-45, 1969.

SKINNER, W. The focused factory. Harvard Business Review, v.52, May-June, p.113-121, 1974.

SLACK, N.; CHAMBERS, S.; HARLAND, C.; HARRISON, A.; JOHNSTON, R. Administração da Produção. São Paulo: Atlas, 1997.

SLACK, N; LEWIS, M. Operations Strategy. Pearson Education, Harlow, 2002. 
TAYLOR, A.; TAYLOR, M. Operations management research: contemporary themes, trends and potential future directions. International Journal of Operations \& Production Management, v.29, n.12, p.1316-1340, 2009.

cross ref

VARGO, S.; LUSCH, R. The four service marketing myths: remnants of a goods-based, manufacturing model. Journal of Service Research, v.6, n.4, p. 324-335, may 2004.

cross

VERMA, R. An empirical analysis of management challenges in service factories, serviceshops, mass services and professional services. International Journal of Service Industry Management, v.11, n.1, p.8-25, 2000.

cross ref

VOSS, C. Alternative paradigm for manufacturing strategy. International Journal of Operations \& Production Management, v.15, n.4, pp.5-16, 1995.

cross ref

WHEELWRIGHT, C.S. Manufacturing strategy: defining the missing link. Strategic Management Journal, v.5, p.6$20,1984$.

ZEITHAML, V.; BITNER, M. Marketing de serviços: a empresa com foco no cliente. 2. ed. Porto Alegre: Bookman, 2003.

\section{Referências Consultadas}

ACUR, N.; BITITCI, U. A balanced approach to strategy process. International Journal of Operations and Production Management, v.24, n.4, p.388-408, 2004.

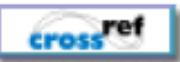

ACUR, N.; ENGLYST, L. Assessment of strategy formulation: how to ensure quality in process and outcome. International Journal of Operations \& Production Management. v. 26, n. 1, p. 69-91, 2006.

cross ref

ANDERSEN, E. S. Toward a Project Management Theory for Renewal Projects. Project Management Journal, v.37, n.4, p.15-30, 2006.

ARANDA, D. A. Service operations strategy, flexibility and performance in engineering consulting firms. International Journal of Operations \& Production Management. v. 23, n. 11, p. 1401-1421, 2003.

cross ref

ARAUJO, L.; SPRINGER, M. Service, products, and institutional structure of production. Industrial Marketing Management. v.35, n.7, p.797-805, 2006.

cross ref

ARMISTEAD, C.; CLARK, G. The “Coping” Capacity Management Strategy in Services and the Influence on Quality Performance. International Journal of Service Industry Management, v. 5, n. 2, p.5-22, 2002.

cross ref

ARMISTEAD, C.; MACHIN, S. Business process management: implications for productivity in multi-stage service networks. International Journal of Service Industry Management, v. 9, n. 4, p. 323-336, 1998.

cross ref

AXELSSON, B., 1992. Corporate strategy models and networks-diverging perspectives. Industrial Networks: A New View of Reality. Routledge, London, p. 184-204, 1992. 
BARCLAY, C.; OSEI-BRYSON, K. Project performance development framework: An approach for developing performance criteria \& measures for information systems (IS) projects. International Journal Production Economics, v. 124, p.272-292, 2010.

cross ref

BOWEN, D.E.; CHASE, R.B.; CUMMINGS, T.G. and et al. Service Management Effectiveness, Jossey-Bass, San Francisco, CA, 1990.

BOWEN, D.E.; LAWLER, E. The empowerment of service workers: what, why, howand when. Sloan Management Review, v. 33, n. 3, 1992.

BRAX, S.A. A manufacturing becoming service provider - challenger and paradox. Management Service Quality, v.15, n.2, p.142-155, 2005.

cross ref

BRIGNALL, T.J., FITZGERALD, L.; JOHNSTON, R.; SILVESTRO, R. Product costing in service organizations. Management Accounting Research, v. 2, p. 227-48, 1991.

cross ref

BUZACOTT, J. A. Service system structure. International Journal Production Economics, v.68, p.15-27, 2000.

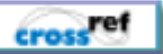

CAMPBELL, A.; LUCHS, S. K. Core Competency-Based Strategy. International Thomson Business Press, 1997.

CHASE, R.B. Where does the customer fit in a service operation. Harvard Business Reviewn, v. 56, n. 4, p. 137-42, 1978.

CORREA, H. L. Flexibilidade nos Sistemas de Produção. Publicado na RAE da FGVSP vol 33 n. 3 , 1993.

CORREA, H. L.; GIANESI, I. G. Gestão Estratégica de Operações de Serviço. Escola Politécnica da USP, Cladea 1995.

FILHO, O. R.; NETO, F. J. Estratégia, Projetos e Processos - uma gestão orquestrada. XXV ENEGEP Porto Alegre, RS, Brasil, 29 de Outubro a 01 de Novembro de 2005.

FITZGERALD, L., JOHNSTON, R., BRIGNALL, T.J., SILVESTRO, R.; VOSS, C. Performance Measurement in Service Businesses. CIMA, 1991.

FITZGERALD, L., JOHNSTON, R., BRIGNALL, T.J., SILVESTRO, R.; VOSS, C. Management Controlin Service Industries. CIMA Research Report, 1990.

FROHLICH, M. T.; DIXON, J. R. A taxonomy of manufacturing strategies revisited. Journal of Operations Management, v.19, p.541-558, 2001.

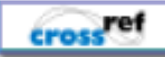

GAGNON, S. Resource-based competition and the new operations Strategy. International Journal of Operations \& Production Management. v.19, n.2, p. 125-138, 1999.

cross ref

GIANESI, I. G. N.; CORREA, H. L. Administração estratégica de serviços. Operações para a satisfação do cliente. São Paulo: Atlas, 1994.

GYAMPAH, A. K.; MEREDITH, J.R.: The operations management research agenda: an update. Journal of Operations Management, v.8, p.250-262, 1989.

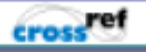

HAYES, H.R.; PISANO, P.G. Beyond world-class the new manufacturing strategy. Harvard Business Review, v.72, n.10, p.77-86, 1994. 
HAYES, H.R.; UPTON, M.D. Operations based strategy. California Management Review, v.40, n.4, p.8-25, 1998.

HAYES, R.H., WHEELWRIGHT, S.C. Restoring our Competitive Edge-Competing Through Manufacturing. Wiley,New York, 1984.

HILL, A. How to organise operations: Focusing or splitting? International Journal Production Economics. v.112, p. 646-654, 2008 .

\section{cross $r$}

HILl, A. V.; COLliER, D. A.; FROEHLE, C. M; GOODALE, J. C.; METTERS, R. D.; VERMA, R. Research opportunities in service process design. Journal of Operations Management, v.20, p.189-202, 2002.

\section{cross ref}

HILL, T.J. Manufacturing Strategy. ed 2. Macmillan, Basingstoke, 1993.

HILL, T.J. Management Strategy: Text and Cases. Irwin, University of Bath, 1989.

JOHNSTON, R. Service operations management: from the roots up. International Journal of Operations \& Production Management. v.25, n. 12, p. 1298-1308, 2005.

cross ref

JOHNSTON, R. Service operations management: return to roots. International Journal of Operations \& Production Management. v.25, n.12, p. 1278-1297, 2005.

cross ref

JOHNSTON, R.; CHAMBERS, S.; HARLAND, C.; HARRISON, A.; SLACK, N. Cases in Operations Management. Pitman Publishing, London, 1993.

JOHNSTON, R.; MORRIS, B. Monitoring and control in service operations. International Journal of Operations and Production Management, vol. 5, p. 32-8, 1985.

cross ref

KHOONG, C. M.; KU, Y. W. The TSC Project: A Strategic R\&D Initiative in Operations Management. International Journal of Operations \& Production Management. v. 14, n. 8, p. 35-46, 1994.

cross ref

KILLEN, C. P.; HUNT, R. A. Dynamic capability through project portfolio management in service and manufacturing industries. International Journal of Managing Projects in Business,v.3, p.157-169, 2010.

cross ref

KOtler, P.; ARMStrong, G. Principles of Marketing. Eighth Edition, Prentice Hall, Englewood Cliffs, New Jersey, 1999.

KRUGMAN, P.R. Plugging in to productivity. US News \& World Report, 1993.

LOVELOCK, C.H. Classifying services to gain strategic marketing insights. Journal of Marketing, v. 47, p. 9-20, 1983.

cross ${ }^{\text {ref }}$

MACHUCA, J. A.; GONZALEZ, M. M.; ESCOBAR, V.G. Service Operations Management research. Journal of Operations Management, v.25, p.585-603, 2006.

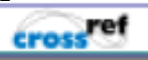

MARTINS, G. S. A Construção do Conhecimento Científico no Campo de Gestão de Operações no Brasil: uma análise sob a ótica de Redes Sociais do período 1997-2008. Fundação Getúlio Vargas, Escola de Adminsitração de Empresas de São Paulo, 2009. 
MAYRING P. Qualitative Inhaltanalyse - Grundlagen und Techniken. Qualitative content analysis. 8th ed. Weinheim, Germany: Beltz Verlag; 2003.

MELLO, C. H.; NETO, P. L.; TURRIONI, J. B. Modelo para o projeto e desenvolvimento de serviços:uma proposta brasileira. In: Simpósio de Engenharia de Produção, XII SIMPEP, 2010, Bauru, SP. Anais... Bauru, SP, 2010.

MILLS, J., PLATTS, K. Manufacturing strategy. International Encyclopedia of Business and Management, v. 4, p. 3126-3141, 1996.

MINTZBERG, H., QUINN, J.B. The Strategy Process: Concepts Contexts Cases. Prentice Hall, Inc.,New Jersey, 1996.

NEELY, A.D. The Servitizations of Manufacturing. An Analyses of Global Trends. In: POMS, College of Service Operations and EurOMa Conference 2007. Londres: London Business School, 2007.

NEELY, A.D.; JARRAR, Y. Extracting value from data - the performance planning value chain. Business Process Management Journal, v.10, n.5, p.506-509, 2004.

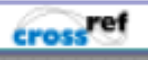

PILKINGTON, A.; FITZGERALD, R. Operations management themes, concepts and relationships: a forward retrospective of IJOPM. International Journal of Operations \& Production Management, v.26, n.11, p.1255-1275, 2006

cross ref

PILKINGTON, A.; LISTON-HEYES, C. Is production and operations management a discipline? A citation/co-citation study'. International Journal of Operations \& Production Management, v.19, n.1, p.7-20, 1999.

cross ref

PILKINGTON, A.; MEREDITH, J. The evolution of the intellectual structure of operations management - 1980-2006: a citation/co-citation analysis. International Journal of Operations Management, v. 27, p.185-202, 2009.

cross ref

PLATTS, K.W.; GREGORY, M.J. Manufacturing audit in the process of strategy formulation. International Journal of Production and Operations Management. v.10, p.5-26, 1990.

cross ref

PLATTS, K.W.; MILLS, J.F.; NEELY, A. D.; GREGORY, M. J.; RICHARDS, A.H. Evaluating manufacturing strategy formulation.processes. International Journal Production Economics, v. 46, p. 233-240, 1996.

cross ${ }^{\text {ref }}$

PORTER, M.E. Toward a dynamic theory of strategy. Fundamental Issues in Strategy: A Research Agenda, Harvard, Business School Press, p. 423-461,1994.

REN, G.; GREGORY, M. Servitization in manufacturing companies: a conceptualization, critical review and research agenda. In: Annual Frontiers in Service Conference, 16. San Francisco, CA, USA, 2007.

ROSS, A.D. Performance-based strategic resource allocation in supply networks. International Journal of Production, Economics, v. 63, p.255-266, 2000.

cross ref

ROTH, A.V.; CHASE, R.B.; VOSS, C. Service in the US. Severn Trent, 1997.

SCHMENNER, R. How can service businesses survive and prosper? Sloan Management Review, Spring, p. 21-32, 1986.

SCHMENNER, R. W. Administração de Operações em Serviços. São Paulo: Futura, 1986. 
SCHMENNER, R. W. Service Firm Location Decisions: Some Midwestern Evidence. International Journal of Service Industry Management, v. 5, n. 2, p. 35-56, 1994.

cross ref

SEURING, S.; MULLER, M. From a literature review to a conceptual framework for sustainable supply chain management. Journal of Cleaner Production,v.16, p.1699-1710, 2008.

cross ref

SHENHAR, A.; DVIR, D. Reinventing Project Management: The Diamond to Successful Growth and Innovation. Boston: Harvard Business School Press, 2007.

SIDHARTHA, R.; CEM C. Designing service processes: a design factor based process model. International Journal Services Technology and Management, v.7, n.1, p.85-107, 2006.

SILVESTRO, R. Positioning services along the volume-variety diagonal. The contingencies of service design, control and improvement. International Journal of Operations \& Production Management,v.19, n.4, p. 399-420, 1999.

cross ref

SKINNER, W. Manufacturing the missing link in corporate strategy. Harvard Business Review, v.47, n.3, p.136-45, 1969.

SKINNER, W. The focused factory. Harvard Business Review, v.52, p.113-121, 1974.

SLACK, N.; CHAMBERS, S.; HARLAND, C.; HARRISON, A.; JOHNSTON, R. Administração da Produção. São Paulo: Atlas, 1997.

SLACK, N; LEWIS, M. Operations Strategy. Pearson Education, Harlow, 2002.

SMITH, T. M.; REECE, J. S. The relationship of strategy, fit, productivity, and business performance in a services setting. Journal of Operations Management, v.17, p.145-161, 1999.

cross ref

TAYLOR, A.; TAYLOR, M. Operations management research: contemporary themes, trends and potential future directions. International Journal of Operations \& Production Management, v.29, n.12, p.1316-1340, 2009.

cross ref

VARGO, S.; LUSCH, R. The four service marketing myths: remnants of a goods-based, manufacturing model. Journal of Service Research, v.6, n.4, p. 324-335, may 2004.

cross ref

VERMA, R. An empirical analysis of management challenges in service factories, service shops, mass services and professional services. International Journal of Service Industry Management, v. 11, n. 1, p. 8-25, 2000.

cross ref

VERMA, R..; GOODALE, J.C. Statistical power in operations management research. Journal of Operations Management, v. 13, n. 2, p. 139-52, 1995.

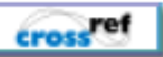

VOSS, C. Alternative paradigm for manufacturing strategy. International Journal of Operations \& Production Management, v.15, n.4, p.5-16, 1995.

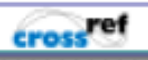

YEEA, C.L.; PLATTS, K.W. A framework and tool for supply network strategy. International Journal Production Economics, v. 104, p. 230-248, 2006.

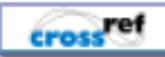


ZEITHAML, V.; BITNER, M. Marketing de serviços: a empresa com foco no cliente. 2. ed. Porto Alegre: Bookman, 2003.

\section{Dados dos autores:}

Nome completo: Juliano Munik

Filiação institucional: Pontifícia Universidade Católica do Paraná - PUCPR

Departamento: Programa de Pós-Graduação em Engenharia de Produção e Sistemas - PPGEPS

Função ou cargo ocupado: Mestrando

Endereço: Rua Imaculada Conceição 1155, Curitiba, Paraná, Brasil, CEP.80215901

Telefone: (41) 32712579

e-mail: juliano.munik@pucpr.br

Nome completo: Edson Pinheiro de Lima

Filiação institucional: Pontifícia Universidade Católica do Paraná - PUCPR

Departamento: Programa de Pós-Graduação em Engenharia de Produção e Sistemas - PPGEPS

Função ou cargo ocupado: Professor

Endereço: Rua Imaculada Conceição 1155, Curitiba, Paraná, Brasil, CEP.80215901

Telefone: (41) 32712579

e-mail: e.pinheiro@pucpr.br

Nome completo: Sérgio Eduardo Gouvêa da Costa

Filiação institucional: Pontifícia Universidade Católica do Paraná - PUCPR

Departamento: Programa de Pós-Graduação em Engenharia de Produção e Sistemas - PPGEPS

Função ou cargo ocupado: Professor

Endereço: Rua Imaculada Conceição 1155, Curitiba, Paraná, Brasil, CEP.80215901

Telefone: (41) 32712579

e-mail: s.gouvea@pucpr.br

Enviado em: 24/08/2011

Aprovado em: 27/04/2012 